\title{
Privacy-preserving schemes for Ad Hoc Social Networks: A survey
}

\author{
Mohamed Amine Ferrag, Leandros Maglaras, Senior Member, IEEE, and Ahmed Ahmim
}

\begin{abstract}
In this paper, we review the state of the art of privacy-preserving schemes for ad hoc social networks, including, mobile social networks (MSNs) and vehicular social networks (VSNs). Specifically, we select and in-detail examine thirty-three privacypreserving schemes developed for or applied in the context of ad hoc social networks. These schemes are published between 2008 and 2016. Based on this existing privacy preservation schemes, we survey privacy preservation models, including location privacy, identity privacy, anonymity, traceability, interest privacy, backward privacy, and content oriented privacy. The recent important attacks of leaking privacy, countermeasures, and game theoretic approaches in VSNs and MSNs are summarized in form of tables. In addition, an overview of recommendations for further research is also provided. With this survey, readers can have a more thorough understanding of research trends in privacy-preserving schemes for ad hoc social networks.
\end{abstract}

\section{Index Terms}

Security, Privacy preservation, Ad hoc social network, Mobile social network, Vehicular social network

\section{INTRODUCTION}

A WIRELESS AD-HOC network consists of mobile platforms which are free to communicate without any central control entity [1]. It can operate in an isolated manner or with fixed networks through gateways. The power of an ad hoc network is that it does not differentiate between a router and a station, i.e., each station contributes to routing. Mobile ad hoc networks (MANETs) and vehicular ad hoc networks (VANETs) are special cases of ad hoc networks. The MANET is an autonomous system of mobile nodes, which has several salient characteristics, namely, dynamic topologies, bandwidth-constrained and energy constrained operation, and limited physical security [2], [3]. The VANET is a special case of MANET, where the mobile nodes are instantiated with vehicles equipped with On-board Unit (OBU) communication devices [4].

Today, in our daily lives, social networking enables us to contact our colleagues, friends, and families through applications such as Facebook, Twitter, Linkedin, Google+, YouTube, and ResearchGate. At the same time, however, ad hoc social networks are getting increasingly important which it takes the human factors into consideration, i.e., human mobility, human selfish status, and human preferences [5], [6], [7], [8]. In this survey, we focus on two types of ad hoc social networks, including, mobile social networks (MSNs) and vehicular social networks (VSNs). Tab. I gives a comparison between ad hoc network (MANET, VANET) and ad hoc social network (MSN, VSN) in terms of node, mobility, connectivity, resource, architecture, scalability, application, typical research issue, and security.

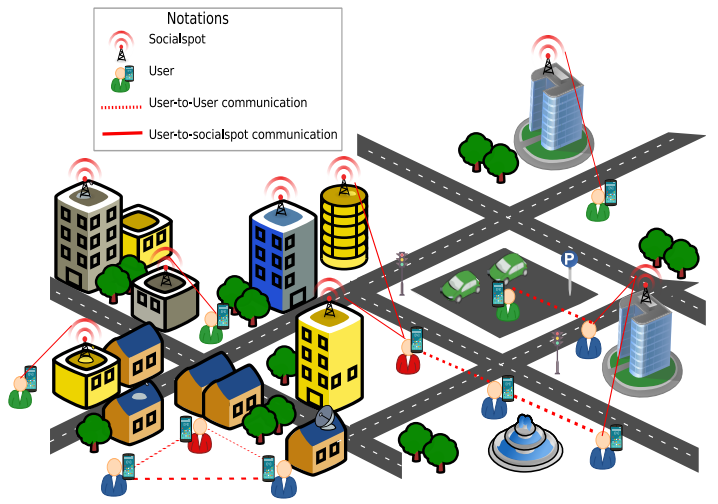

(a) Mobile social networks (MSN)

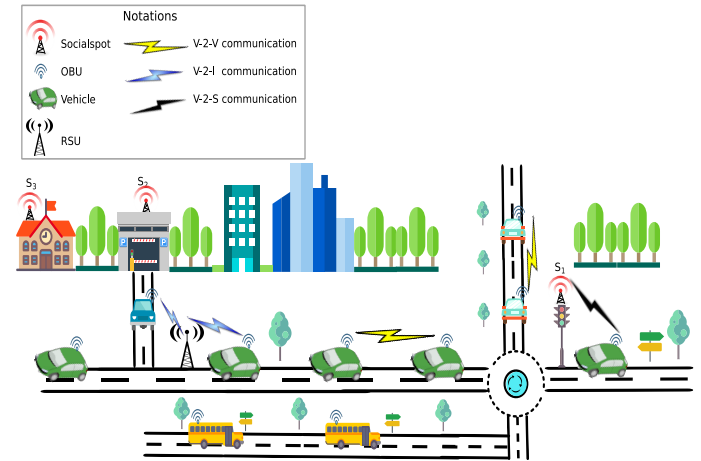

(b) Vehicular social networks (VSN)

Fig. 1. A global architecture of Ad Hoc Social Network

M. A. Ferrag is with Department of Computer Science, Guelma University, Algeria e-mail: mohamed.amine.ferrag@gmail.com phone: +213661-873-051

L. Maglaras is with School of Computer Science and Informatics, Cyber Security Centre, De Montfort University, Leicester, UK e-mail: leandros.maglaras@dmu.ac.uk

A. Ahmim is with Department of Mathematics and Computer Science, University of Larbi Tebessi, Algeria e-mail: a.ahmim@gmail.com

Manuscript received 2016 
TABLE I

COMPARISON OF NETWORK CHARACTERISTICS

\begin{tabular}{|c|c|c|c|c|}
\hline & \multicolumn{2}{|l|}{ Ad hoc network } & \multicolumn{2}{|l|}{ Ad hoc social network } \\
\hline & MANET & VANET & MSN & VSN \\
\hline Node & $\begin{array}{l}\text { Laptop, } \\
\text { smartphone, pocket } \\
\text { PC, router...etc }\end{array}$ & Vehicles & Human & $\begin{array}{l}\text { Vehicles with social } \\
\text { properties }\end{array}$ \\
\hline Mobility & Random & On-Road & Human mobility & $\begin{array}{ll}\text { On-Road } & \text { with } \\
\text { socialspot } & \end{array}$ \\
\hline Connectivity & Random & $\begin{array}{l}\text { Random and Inter- } \\
\text { mittent }\end{array}$ & Interest social & Interest social \\
\hline Resource & $\begin{array}{l}\text { Limited hardware } \\
\text { and power limited } \\
\text { by battery }\end{array}$ & Almost unlimited & $\begin{array}{l}\text { Limited hardware and } \\
\text { power limited by bat- } \\
\text { tery }\end{array}$ & Almost unlimited \\
\hline Architecture & Node-to-node & $\begin{array}{l}\text { Vehicle-to-vehicle, } \\
\text { Vehicle-to-RSU }\end{array}$ & $\begin{array}{l}\text { Node-to-node, Node- } \\
\text { to-socialspot }\end{array}$ & $\begin{array}{l}\text { Vehicle-to-vehicle, } \\
\text { Vehicle-to-RSU, } \\
\text { Vehicle-to-socialspot }\end{array}$ \\
\hline Scalability & 50-100 nodes & Huge & $50-100$ nodes & Huge \\
\hline Application & $\begin{array}{l}\text { Military, disaster } \\
\text { (specific) }\end{array}$ & $\begin{array}{l}\text { Safety, traffic, pay- } \\
\text { ment }\end{array}$ & $\begin{array}{l}\text { Mobile social } \\
\text { applications, location- } \\
\text { based applications }\end{array}$ & $\begin{array}{l}\text { Vehicular social } \\
\text { applications, location- } \\
\text { based applications }\end{array}$ \\
\hline $\begin{array}{l}\text { Typical } \\
\text { Research Issue }\end{array}$ & Routing & Application & Application & Application \\
\hline Security & Sensitive & Sensitive & Highly-sensitive & Highly-sensitive \\
\hline
\end{tabular}

TABLE II

PUBLICATION DATE BREAKDOWN - SURVEYED PAPERS

\begin{tabular}{|c|c|c|c|c|c|}
\hline Paper & Year & Paper & Year & Paper & Year \\
\hline [9] & 2008 & [10] & 2011 & [11] & 2013 \\
\hline 12 & 2010 & 13 & 2011 & 14 & 2014 \\
\hline $\mid 15$ & 2010 & $\mid 16$ & 2011 & |17| & 2014 \\
\hline $\mid 18$ & 2010 & 19 & 2012 & $|20|$ & 2014 \\
\hline 21 & 2010 & $\mid 22$ & 2012 & |23 & 2015 \\
\hline |24] & 2010 & |25] & 2012 & 26 & 2015 \\
\hline 27] & 2011 & $\mid 28$ & 2012 & $\mid 29$ & 2016 \\
\hline |30| & 2011 & $|31|$ & 2012 & [32] & 2016 \\
\hline |33 & 2011 & $\mid 34$ & 2012 & |35| & 2016 \\
\hline [36 & 2011 & (37) & 2013 & [38] & 2016 \\
\hline $\mid 39$ & 2011 & |40| & 2013 & [41] & 2016 \\
\hline
\end{tabular}

As shown in Fig 1 a, MSN is composed of a set of mobile users $U=\left\{u_{1}, \cdots, u_{n}\right\}$ with some socialspots $S=\left\{s_{1}, \cdots, s_{n}\right\}$ in a city environment, which each user $u_{i}$ having equal communication range $R_{u_{i}}$ [5], [7], [8]. Similarly to MSN, as shown in Fig 1 b, a VSN is composed of a large number of vehicles $V=\left\{v_{1}, \cdots, v_{n}\right\}$ equipped with on-board units (OBUs), Roadside Units (RSUs) and some socialspots $S=\left\{s_{1}, \cdots, s_{n}\right\}$. Using the communication capabilities of their OBUs, the vehicles can communicate with each other, as well as with RSUs and socialspot $s_{i}$, i.e., vehicle-to-vehicle (V-2-V) communication, vehicle-to-infrastructure (V-2-I) communication, vehicle-to-socialspot (V-2-S) communication [6], [42]. These three types of communications can happen using the wireless access technologies that are available today such as cellular systems $(3 \mathrm{G} / 4 \mathrm{G} / 5 \mathrm{G})$, WLAN/Wi-Fi, WiMAX, and DSRC/WAVE [43].

Since the social characteristics are integrated into ad hoc networks, MAN and VSN have become very sensitive to security and privacy compared with MANET and VANET. In other words, security issue is crucial to the full adoption of MSN and VSN, especially for the privacy. Based on identities, pseudonyms, locations, and profiles, an adversary can launch active or passive attacks (deliberately delays, drops, corrupts, or modifies messages) in order to steal the social data as well as to damage the V-2-V, V-2-I, and V-2-S communications. Hence, in order to protect the social community, the privacy-preserving schemes employed in MSNs and VSNs should satisfy the following security requirements: authentication, integrity, non-repudiation, access control, and privacy [44].

In a recent survey paper which was published in 2016 [45] authors review the state of the art of privacy preserving techniques for online social networks (OSNs). In addition, other recent surveys in [46] and [42] published in 2015 provide information about applications, platforms, system architectures in MSNs and VSNs, respectively. Neither of the published, state-of-the-art literature provide a comprehensive survey for recent advances in privacy-preserving schemes for MSNs and VSNs. The aim of this survey paper is to provide comprehensive and systematic review of the recent studies on published privacy-preserving schemes for ad hoc social networks, including, MSNs and VSNs. More precisely, we select and in-detail examine thirty-three privacy-preserving schemes. See Tab. II for a breakdown of publication dates and Fig. 2 for the properties investigated.

The main contributions of this paper are: 


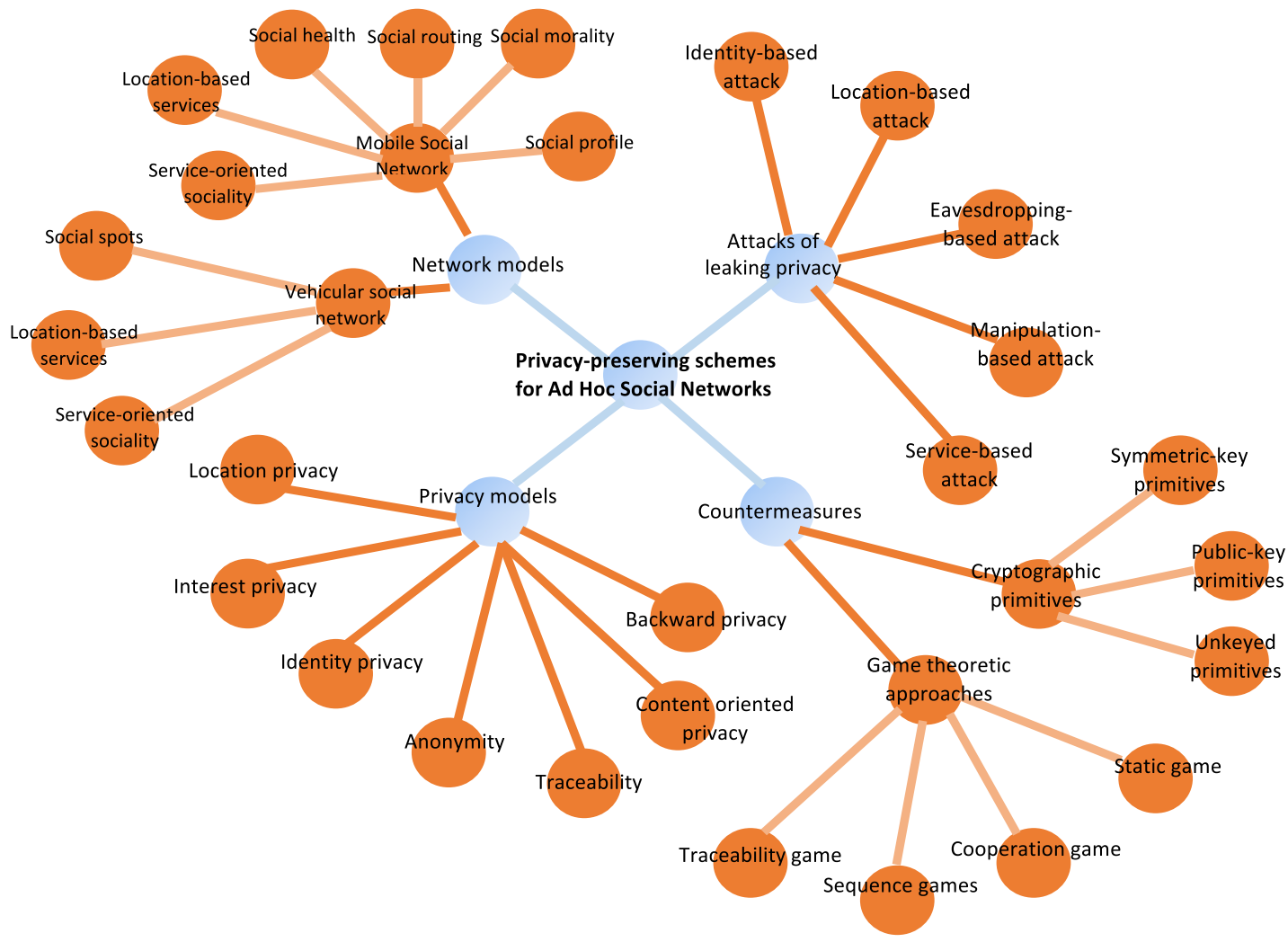

Fig. 2. Organization of surveyed papers

- We present various privacy preservation models for MSNs and VSNs, including, location privacy, identity privacy, anonymity, traceability, interest privacy, backward privacy, and content oriented privacy.

- We provide a classification for the attacks of leaking privacy for MSNs and VSNs, including, identity-based attacks, location-based attacks, eavesdropping-based attacks, manipulation-based attacks, and service-based attacks.

- We present various countermeasures and game theoretic approaches proposed for MSNs and VSNs.

- We present a side-by-side comparison in a tabular form for the current state-of-the-art of privacy-preserving schemes (thirty-three) proposed for MSNs and VSNs.

- We present a discussion of recommendations for further research, namely, including, privacy preserving methods, interdependent privacy, combination of privacy metrics, and identification of areas of vulnerability.

The remainder of this paper is organized as follows. Section II presents various privacy preservation models for MSNs and VSNs. In Section III] we provide a classification for the attacks of leaking privacy for MSNs and VSNs. Section IV] presents various countermeasures and game theoretic approaches proposed for MSNs and VSNs. In sections V $\mathrm{V}$ and VI, we present a side-by-side comparison in a tabular form for the current state-of-the-art of privacy-preserving schemes proposed for MSNs and VSNs, respectively. Finally, we discuss open issues and recommendations for further research in Section VII and draw our conclusions in Section VIII.

TABLE III

PRIVACY-PRESERVING MODELS

\begin{tabular}{|c|c|c|}
\hline Reference & Data protected & Model \\
\hline 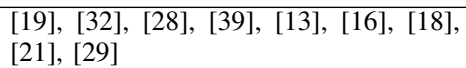 & Location & Location privacy \\
\hline 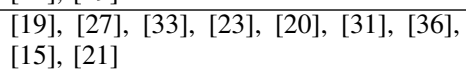 & ID & Identity privacy \\
\hline$|37|,|34|,|11|, \mid 39],[10], \mid 15], \mid 16]$ & ID location & Anonymity \\
\hline$[47],[48],[49],[20],[31],[36]$ & ID & Traceability \\
\hline$[21],[30]$ & Interest & Interest privacy \\
\hline $\mid 15$ & Backward & Backward privacy \\
\hline [40], |35], [41] & All data in the network & Content oriented privacy \\
\hline$[14 \mid,[30]$ & All data in the network & Trust evaluation \\
\hline
\end{tabular}




\section{PRIVACY PRESERVATION MODELS}

As shown in Tab. III the papers we review are all related with privacy preservation in ad hoc social networks, and could be divided into location privacy, identity privacy, anonymity, traceability, interest privacy, backward privacy, and content oriented privacy.

\section{A. Location privacy}

Location privacy is one of the most important models for privacy in VSNs and MSNs, since the place of equipment (mobile phone or vehicle) can be linked to the owners. If a privacy-preserving scheme cannot guarantee the location privacy, users will be skeptical and it cannot be accepted by the public. However, there are many solutions to ensure the location privacy in MSNs and VSNs.

In MSNs, there are two papers dealing the location privacy [19], [32]. Liang et al. [19] proposes a proximity measurement with morality-driven data forwarding. This method provides the location privacy by mixing the hotspot based on multiplying a subgroup element. Li et al. [32] analyzes the disclosed locations [50] in the MSN applications and proposes a system-level privacy control approach. This approach, via the decision tree model, it provides the privacy of location sharing in MSN.

In VSNs, there are seven papers dealing the location privacy [28], [39] [13], [16], [18], [21], [29]. Lu et al. [28] proposes a strategy for the location privacy based on pseudonym self-delegated generation with conditional tracking. With this strategy, when a vehicle changes its pseudonyms, the location privacy can be guaranteed. In the paper [39], the location privacy is guaranteed using Lite-CA-based public key cryptosystem. According to Lin et al. [13], achieving receiver-location privacy preservation can be guaranteed by the social-tier dissemination phase, where the social tier request vehicles to help forwarding the packet to its neighboring social spots, and it shouldn't degrade the packet delivery performance. Lu et al. [16] proposes another technique based on pseudonyms changing at small social spot and large social spot. This technique, via anonymity analysis, can provide a promising solution for the location privacy. Related to the method proposed from Lu et al. [16], the author proposes an idea which vehicles periodically change multiple pseudo-IDs [21]. Lu et al. [18] proposes another idea called "Sacrificing the Plum Tree for the Peach Tree". This idea is a technique based on a collection of social spots for protecting receiver-location privacy and improving the performance of packet forwarding. Yu et al. [29] proposes a scheme based on a collection of social spots, including 1) global social spot and 2) individual social spot. These two types of social spot exploit the meeting opportunities for pseudonym changing in order to improve the location privacy.

\section{B. Identity privacy}

Before ensuring the location privacy, it is necessary to reassure the identity privacy. This can be acheived if in order to place of real identity, each equipment uses a pseudonym. There are many solutions which are based on concealing the real identity to ensure the identity privacy in MSNs and VSNs.

In MSNs, there are four papers dealing the identity privacy [19], [27], [33], [23]. Note that there are techniques that provide both identity and location privacy such as multiple-pseudonym technique [19]. Lu et al. [27] proposes an algorithm, called Patient Joining, for achieving the real identity privacy by a pseudo-id. This algorithm is executed between the trusted authority and patient and it outputs a pseudo-id. Liang et al. [33] addresses the identity privacy in an emergency situation by ensuring unlinkability of the transactions and enhancing availability. Especially, in the emergency call generation phase, the user identity is guaranteed using a group signature proposed in [51]. Zhang et al. [23] develop a personalized fine-grained filtering for the identity privacy. This filtering is based on social-assisted filter distribution.

In VSNs, there are five papers dealing the identity privacy [20], [31], [36], [15], [21]. Chim et al. [20] support privacypreserving of the drivers. The identity privacy is preserved using two ideas, including 1) the idea of pseudo-identity and 2) the idea of anonymous credential. Liang et al. [31] proposes an idea based on a tamper-proof device activation password, which the RSU uses in order to verify the vehicle's identity and sends its tamper-proof device an anonymous credential. The trusted authority can reveal the real identity pf the vehicle to a third party for billing purposes. Chim et al. [36] proposes a scheme that uses a different pseudo identity for each session. This scheme preserves the real identity based on the handshaking phase, which is executed between RSU and the trusted authority. Sun et al. [15] analyzes the identity revocation based on the certificate revocation list in order to exclude an unexpired membership. Based on the work presented in [20], Liang et al. [31] presents the idea of pseudo-identity for ensuring identity privacy. Aiming to reduce the linkage between the identity and location of vehicles, Yan et al. [52] propose a scheme that enhances the privacy using the idea of cell-based communication. Authors view vehicular networks as consisting of non-overlapping sub-networks restricted to a geographic area referred to as a cell. Each cell has a server that maintain a list of pseudonyms that can be assigned to the vehicles. Although the idea of dividing the network is interesting and has been extensively used for clustering [53] and routing reasons, the existence of a server in each area increases the cost of the solution and also makes it infeasible. 


\section{Anonymity- Untraceability}

Anonymity is an important security aspect of wireless communications, since it not only protects the privacy of the users but also reduces the chances of attacks based on impersonation [54]. Untraceability is a related issue to anonymity, since if a user is traceable, its hidden identity can be revealed through the profiling of user's activity. Note that most of the anonymity schemes use a public key infrastructure (PKI) [10]. For evaluating anonymity and protecting privacy, Sweeney et al. [55] proposes the k-anonymity mode. Other interesting methods which are presented in the papers [56], [57], similar to the model in [55], introduce the notions of sender and receiver k-anonymity. Specifically, Wang et al. [57] proposes a protocol, which ensures the anonymous transmission in a Local Ring. Moreover, there is a recent work presented in the paper [37] that proposes a profile matching protocol in MSNs, called PPM, for ensuring the anonymity (from conditional to full). The PPM protocol uses three approaches, including, 1) explicit comparison-based approach, 2) implicit comparison-based approach, and 3) implicit predicate-based approach. In addition, the PPM protocol uses two anonymity enhancing techniques, including, 1) anonymity measurement and 2) anonymity enhancement.

In VSNs, there are six papers dealing the anonymity [34], [11], [39], [10], [15], [16]. Xiong et al. [34] proposes a protocol that supports multi-level anonymity using the ring signature that was initialy presented in [58] and [59]. Based on a pseudo-ID and anonymous certificate, Ying et al. [11] proposes an idea to provide the driver with a satisfactory degree of anonymity. In the paper [39], the anonymity is guaranteed using the technique of on-path onion encryption. For generating pseudonyms, Huang et al. [10] proposes an anonymity scheme, called PACP. The proposed PACP scheme is effective and efficient compared with the anonymity schemes presented in the papers [60], [4] in terms of latency. Sun et al. [15] proposes pseudonymous authentication scheme for the conditional anonymity, which is preserved by three techniques, including, 1) pseudonymous authentication, 2) anonymous authentication for certificate updating, and 3) certificate updating based on re-signature technology. The paper [16] analysis the Quality of Privacy (QoP) with the proposal of two anonymity analytic models, including, 1) anonymity analysis on pseudonym changing at a small social spot (such as the road intersection), and 2) anonymity analysis on pseudonym changing at a large social spot (such as the free parking lot).

\section{Traceability}

Traceability is a very important property, where the trusted authority is able to trace a node that is misbehaving in the network. As discussed in the anonymity sub section, we have both the conditional traceability on signature and the full traceability on signature [61], [51]. To the best of our knowledge, there is no current work studying the traceability in MSN, but we suggest the two works presented in the papers [47], [48], [49] for possible applicability on MSNs. Ni et al. [47] proposes a protocol, called AMA, for carpooling systems. The AMA protocol supports anonymity and traceability based on the trace phase, which consists in calculating the public key of the passenger (driver) after the carpooling trip. Sun et al. [48] proposes an architecture, called SAT, for achieving the traceability based on the blind signature [62], [63]. The SAT architecture is an improvement of the idea presented in [49]. Unlike the MSN networks, there are other recently proposed works that aim in preserving traceability in VSNs [20], [31], [36]. Chim et al. [20], via non-repudiation property of messages, address the traceability based on the real identity of a particular vehicle, where the trusted authority can retrieve the real identity. Lu et al. [31] proposes an idea based on use self-generated pseudonyms instead of real-world IDs. Chim et al. [36] propose a scheme based the real identity tracking and revocation phase for satisfied the traceability and revocability.

\section{E. Interest privacy}

Since the nodes in ad hoc social networks are formed based on a common interest, the privacy of these common interests should be preserved. The interest privacy has been explored firstly in the paper [21]. The topics of common interest in the paper [21] focuses on like-minded vehicles to chat. More precisely, Lu et al. in [21] proposes a protocol, called FLIP, which is based on authenticated key exchange (AKE) protocols [64]. Based on degree of interest verification, Rabieh et al. [26] uses the attribute based encryption in order to preserve the interest privacy.

\section{F. Backward privacy}

Once the nodes in ad hoc social network have been revoked, they should reveal no information in the revocation period. There is one recently proposed work in [15] that discusses the backward privacy in VSNs. Sun et al. [15] propose an authentication scheme called PASS for preserving the backward privacy. The PASS scheme use the one-way hash function, which it is still difficult for any entity to reduce the certificate revocation used by the revoked vehicle. Moreover, there are some works related to the backward privacy models, such as the forward secrecy and the backward secrecy [25].

\section{G. Content oriented privacy}

As discussed in the papers [40], [35], the content oriented privacy is based on three properties, including 1) immutability, 2) transparency, and 3) accountability. Ferrag et al. [40] proposes a scheme called ECPDR, which is based on node certificate 
updating. Based an idea of certificate evolution, Ferrag et al. [17] proposes a scheme called SDPP. Ferrag et al. in [35] proposes another scheme called EPSA. The EPSA scheme uses the short signatures technique and the public key encryption with keyword search for ensuring content oriented privacy. There are also other works related to the content oriented privacy models, such as the impersonator resistance [27], [17] and the trust evaluation [14], [30].

TABLE IV

SUMMARY OF PRIVACY ATTACKS IN MSNS AND DEFENSE SCHEMES

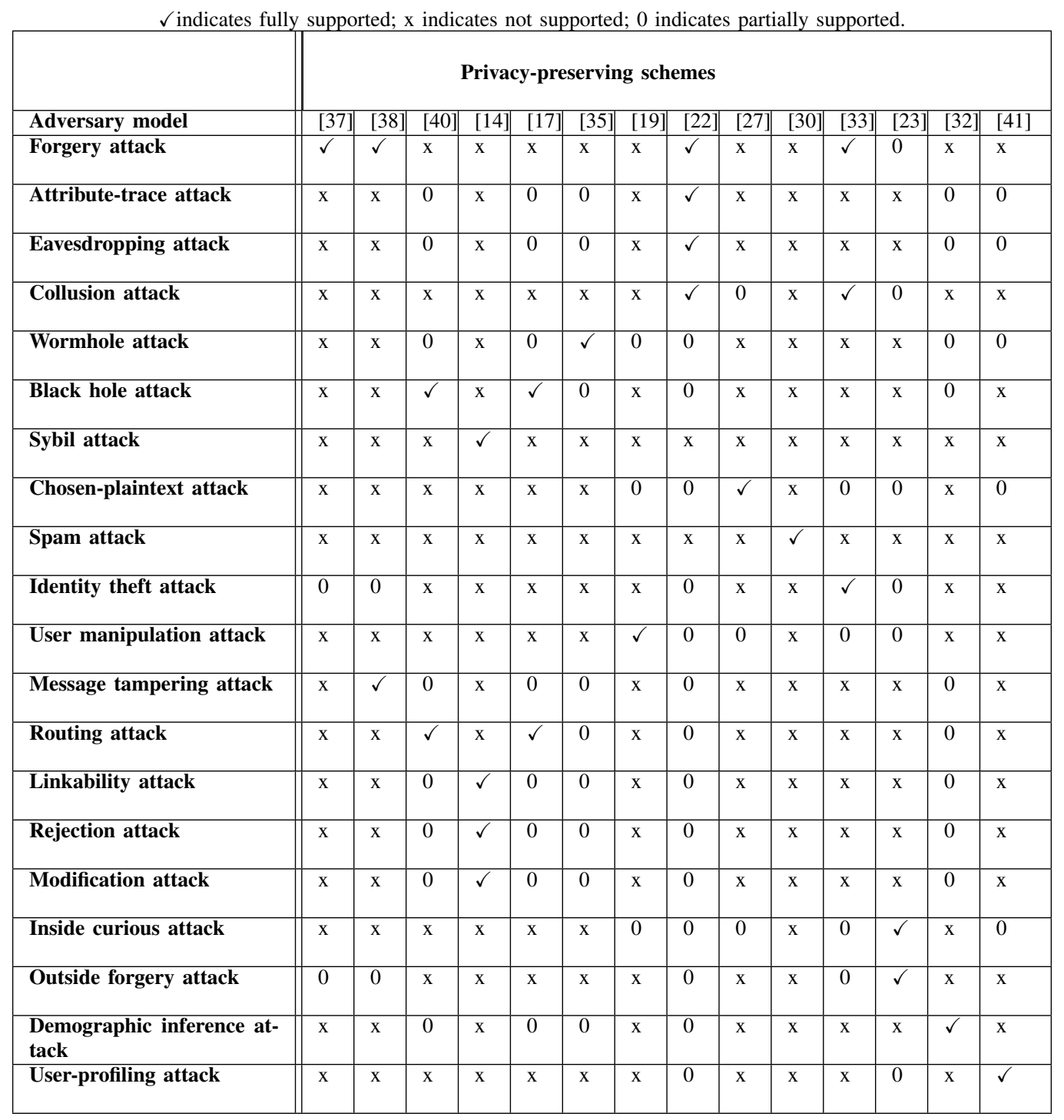

\section{ATTACKS OF LEAKING PRIVACY}

In this section, we discuss the attacks of leaking privacy in Ad Hoc Social Networks. The classification of attacks in ad hoc networks frequently mentioned in literature is done using different criteria such as passive or active, internal or external [65], [66], [67], [68] etc. In our survey articvle we classify the attacks of leaking privacy in five categories as shown in Fig. 3 including, 1) identity-based attack, 2) location-based attack, 3) eavesdropping-based attack, 4) manipulation-based attack, and 5) service-based attack. In addition, Tab. IV and Tab. V give a detailed summary of security threats in MSNs and VSNs, respectively.

\section{A. Identity-based attack}

The attacks that belong to this category are somehow related to the manipulation of the identity of legitimate users. As identity based attacks we can characterize the Sybil attack and the Impersonation attack.

- Sybil attack: When an adversary node has multiple identities, a Sybil attack can be launched in the ad hoc social network. The major goal of the adversary in this attack is to be a destination repeatedly. Once the packets are routed to him, then 
TABLE V

SUMMARY OF PRIVACY ATTACKS IN VSNS AND DEFENSE SCHEMES

\begin{tabular}{|c|c|c|c|c|c|c|c|c|c|c|c|c|c|c|c|c|c|c|}
\hline \multicolumn{19}{|c|}{ Privacy-preserving schemes } \\
\hline Adversary model & 25 & 28 & [12 & [20] & 31 & [34 & 111 & [36] & [39] & [10] & 15 & 13 & 【16] & [18 & [21] & [24] & [9] & 29 \\
\hline Sybil attack & $\checkmark$ & $\mathrm{x}$ & $\checkmark$ & $\mathrm{x}$ & $\checkmark$ & 0 & $\mathrm{x}$ & 0 & $\mathrm{x}$ & 0 & $\mathrm{x}$ & 0 & $\mathrm{x}$ & $\mathrm{x}$ & $\mathrm{x}$ & $\mathrm{x}$ & 0 & 0 \\
\hline Global external attack & $\mathrm{x}$ & $\checkmark$ & $\mathrm{x}$ & $\mathrm{x}$ & 0 & $\mathrm{x}$ & 0 & $\mathrm{x}$ & $\mathrm{x}$ & $\mathrm{x}$ & $\mathrm{x}$ & $\mathrm{x}$ & $\mathrm{x}$ & $\mathrm{x}$ & $\mathrm{x}$ & $\mathrm{x}$ & $\mathrm{x}$ & 0 \\
\hline Replaying attack & $\mathrm{x}$ & $\mathrm{x}$ & $\checkmark$ & $\checkmark$ & $\mathrm{x}$ & $\checkmark$ & $\mathrm{x}$ & $\checkmark$ & $\mathrm{x}$ & $\checkmark$ & $\checkmark$ & $\mathrm{x}$ & $\mathrm{x}$ & $\mathrm{x}$ & $\mathrm{x}$ & $\mathrm{x}$ & $\checkmark$ & $\checkmark$ \\
\hline Impersonation attack & $\mathrm{x}$ & $\mathrm{x}$ & 0 & $\mathrm{x}$ & 0 & $\checkmark$ & $\mathrm{x}$ & $\checkmark$ & $\mathrm{x}$ & $\checkmark$ & $\mathrm{x}$ & $\checkmark$ & $\mathrm{x}$ & $\mathrm{x}$ & $\mathrm{x}$ & $\mathrm{x}$ & $\checkmark$ & $\checkmark$ \\
\hline Location tracking attack & $\mathrm{x}$ & 0 & $\mathrm{x}$ & $\mathrm{x}$ & $\checkmark$ & $\mathrm{x}$ & 0 & $\mathrm{x}$ & $\mathrm{x}$ & $\mathrm{x}$ & $\mathrm{x}$ & $\mathrm{x}$ & $\mathrm{x}$ & $\mathrm{x}$ & $\mathrm{x}$ & $\mathrm{x}$ & $\mathrm{x}$ & 0 \\
\hline Identity revealing attack & 0 & $\mathrm{x}$ & 0 & $\mathrm{x}$ & $\checkmark$ & 0 & $\mathrm{x}$ & 0 & $\mathrm{x}$ & 0 & $\mathrm{x}$ & 0 & $\mathrm{x}$ & $\mathrm{x}$ & $\mathrm{x}$ & $\mathrm{x}$ & 0 & 0 \\
\hline Eavesdropping attack & $\mathrm{x}$ & $\mathrm{x}$ & $\mathrm{x}$ & $\mathrm{x}$ & $\mathrm{x}$ & $\mathrm{x}$ & $\checkmark$ & $\mathrm{x}$ & $\mathrm{x}$ & $\checkmark$ & $\mathrm{x}$ & $\mathrm{x}$ & $\checkmark$ & 0 & 0 & 0 & $\checkmark$ & 0 \\
\hline Forgery attack & $\mathrm{x}$ & 0 & $\mathrm{x}$ & $\mathrm{x}$ & 0 & $\mathrm{x}$ & $\checkmark$ & $\mathrm{x}$ & $\mathrm{x}$ & $\mathrm{x}$ & $\mathrm{x}$ & $\mathrm{x}$ & $\mathrm{x}$ & $\mathrm{x}$ & $\mathrm{x}$ & $\mathrm{x}$ & $\mathrm{x}$ & 0 \\
\hline Chosen plaintext attack & $\mathrm{x}$ & $\mathrm{x}$ & $\mathrm{x}$ & $\mathrm{x}$ & $\mathrm{x}$ & $\mathrm{x}$ & $\mathrm{x}$ & $\mathrm{x}$ & $\checkmark$ & $\mathrm{x}$ & $\mathrm{x}$ & $\mathrm{x}$ & $\mathrm{x}$ & $\mathrm{x}$ & $\mathrm{x}$ & $\mathrm{x}$ & $\mathrm{x}$ & 0 \\
\hline Chosen ciphertext attack & $\mathrm{x}$ & $\mathrm{x}$ & $\mathrm{x}$ & $\mathrm{x}$ & $\mathrm{x}$ & $\mathrm{x}$ & $\mathrm{x}$ & $\mathrm{x}$ & $\checkmark$ & $\mathrm{x}$ & $\mathrm{x}$ & $\mathrm{x}$ & $\mathrm{x}$ & $\mathrm{x}$ & $\mathrm{x}$ & $\mathrm{x}$ & $\mathrm{x}$ & 0 \\
\hline $\begin{array}{l}\text { Adaptive chosen cipher- } \\
\text { text attack }\end{array}$ & $\mathrm{x}$ & $\mathrm{x}$ & $\mathrm{x}$ & $\mathrm{x}$ & $\mathrm{x}$ & $\mathrm{x}$ & $\mathrm{x}$ & $\mathrm{x}$ & $\checkmark$ & $\mathrm{x}$ & $\mathrm{x}$ & $\mathrm{x}$ & $\mathrm{x}$ & $\mathrm{x}$ & $\mathrm{x}$ & $\mathrm{x}$ & $\mathrm{x}$ & 0 \\
\hline $\begin{array}{l}\text { Man-in-the-middle } \\
\text { attack }\end{array}$ & $\mathrm{x}$ & $\mathrm{x}$ & $\mathrm{x}$ & $\mathrm{x}$ & $\mathrm{x}$ & $\mathrm{x}$ & $\mathrm{x}$ & $\mathrm{x}$ & $\checkmark$ & $\mathrm{x}$ & $\mathrm{x}$ & $\mathrm{x}$ & $\mathrm{x}$ & $\mathrm{x}$ & $\mathrm{x}$ & $\mathrm{x}$ & $\mathrm{x}$ & 0 \\
\hline $\begin{array}{ll}\begin{array}{l}\text { Denial-of-service } \\
\text { attack }\end{array} & \\
\text { anoS })\end{array}$ & $\mathrm{x}$ & $\mathrm{x}$ & 0 & 0 & $\mathrm{x}$ & 0 & $\mathrm{x}$ & 0 & $\mathrm{x}$ & 0 & $\checkmark$ & $\mathrm{x}$ & $\mathrm{x}$ & $\mathrm{x}$ & $\mathrm{x}$ & $\mathrm{x}$ & 0 & 0 \\
\hline Source bogus attack & $\mathrm{x}$ & $\mathrm{x}$ & 0 & 0 & $\mathrm{x}$ & 0 & $\mathrm{x}$ & 0 & $\mathrm{x}$ & 0 & 0 & $\checkmark$ & $\mathrm{x}$ & $\mathrm{x}$ & $\mathrm{x}$ & $\mathrm{x}$ & 0 & 0 \\
\hline Black/grey hole attack & $\mathrm{x}$ & $\mathrm{x}$ & $\mathrm{x}$ & $\mathrm{x}$ & $\mathrm{x}$ & $\mathrm{x}$ & 0 & $\mathrm{x}$ & $\mathrm{x}$ & 0 & $\mathrm{x}$ & $\checkmark$ & 0 & $\checkmark$ & 0 & $\checkmark$ & 0 & $\mathrm{x}$ \\
\hline $\begin{array}{l}\text { Successive-response } \\
\text { attack }\end{array}$ & $\mathrm{x}$ & $\mathrm{x}$ & $\mathrm{x}$ & $\mathrm{x}$ & $\mathrm{x}$ & $\mathrm{x}$ & 0 & $\mathrm{x}$ & $\mathrm{x}$ & 0 & $\mathrm{x}$ & $\mathrm{x}$ & 0 & 0 & $\checkmark$ & 0 & 0 & $\mathrm{x}$ \\
\hline Packet analysis attack & $\mathrm{x}$ & $\mathrm{x}$ & $\mathrm{x}$ & $\mathrm{x}$ & $\mathrm{x}$ & $\mathrm{x}$ & 0 & $\mathrm{x}$ & $\mathrm{x}$ & 0 & $\mathrm{x}$ & $\mathrm{x}$ & 0 & 0 & 0 & $\checkmark$ & 0 & $\mathrm{x}$ \\
\hline Packet tracing attack & $\mathrm{x}$ & $\mathrm{x}$ & $\mathrm{x}$ & $\mathrm{x}$ & $\mathrm{x}$ & $\mathrm{x}$ & 0 & $\mathrm{x}$ & $\mathrm{x}$ & 0 & $\mathrm{x}$ & $\mathrm{x}$ & 0 & 0 & 0 & $\checkmark$ & 0 & $\mathrm{x}$ \\
\hline $\begin{array}{l}\text { Brute force cryptanalytic } \\
\text { attack }\end{array}$ & $\mathrm{x}$ & $\mathrm{x}$ & $\mathrm{x}$ & $\mathrm{x}$ & $\mathrm{x}$ & $\mathrm{x}$ & $\mathrm{x}$ & $\mathrm{x}$ & 0 & $\mathrm{x}$ & $\mathrm{x}$ & $\mathrm{x}$ & $\mathrm{x}$ & $\mathrm{x}$ & $\mathrm{x}$ & $\mathrm{x}$ & $\mathrm{x}$ & $\checkmark$ \\
\hline Incorrect data attack & $\mathrm{x}$ & $\mathrm{x}$ & $\mathrm{x}$ & $\mathrm{x}$ & $\mathrm{x}$ & $\mathrm{x}$ & $\mathrm{x}$ & $\mathrm{x}$ & 0 & $\mathrm{x}$ & $\mathrm{x}$ & $\mathrm{x}$ & $\mathrm{x}$ & $\mathrm{x}$ & $\mathrm{x}$ & $\mathrm{x}$ & $\mathrm{x}$ & $\checkmark$ \\
\hline Liability attack & $\mathrm{x}$ & $\mathrm{x}$ & $\mathrm{x}$ & $\mathrm{x}$ & $\mathrm{x}$ & $\mathrm{x}$ & $\mathrm{x}$ & $\mathrm{x}$ & 0 & $\mathrm{x}$ & $\mathrm{x}$ & $\mathrm{x}$ & $\mathrm{x}$ & $\mathrm{x}$ & $\mathrm{x}$ & $\mathrm{x}$ & $\mathrm{x}$ & $\checkmark$ \\
\hline
\end{tabular}

he can realize other types of attacks such as the selective forwarding attack [69]. The TSE system [14] can resist the sybil attacks using the idea of multiple reviews in a short time period. The scheme in [12] can post detected the Sybil attacks by multiple valid pseudo-IDs. The framework in [31] can detect the Sybil attacks using the ID-based signature and the ID-based online/offline signature.

- Impersonation attack: During the registration phase, when the vehicle with real identity generates its pseudo identity, an impersonation adversary records this pseudo identity, which he can realize other types of attacks such as the identity revealing attack [31] and the identity theft attack [33]. The protocol in [34], the PACP protocol in [10], and the SECSPP scheme in [9] use authentication of messages to guard against impersonation attacks. The SPECS schemes in [36] uses a phase called real identity tracking and revocation against an impersonation adversary. Since the single-attribute encryption is employed in the STAP protocol [13], an impersonation adversary can be detected. With the use the safety message and include a valid certificate from the trusted register authority, the MixGroup scheme in [29] can avoid the impersonation attacks.

\section{B. Location-based attack}

This category of attacks is based on revealing the user location, and it consists of two major attacks the forgery attack and the global external attack. 


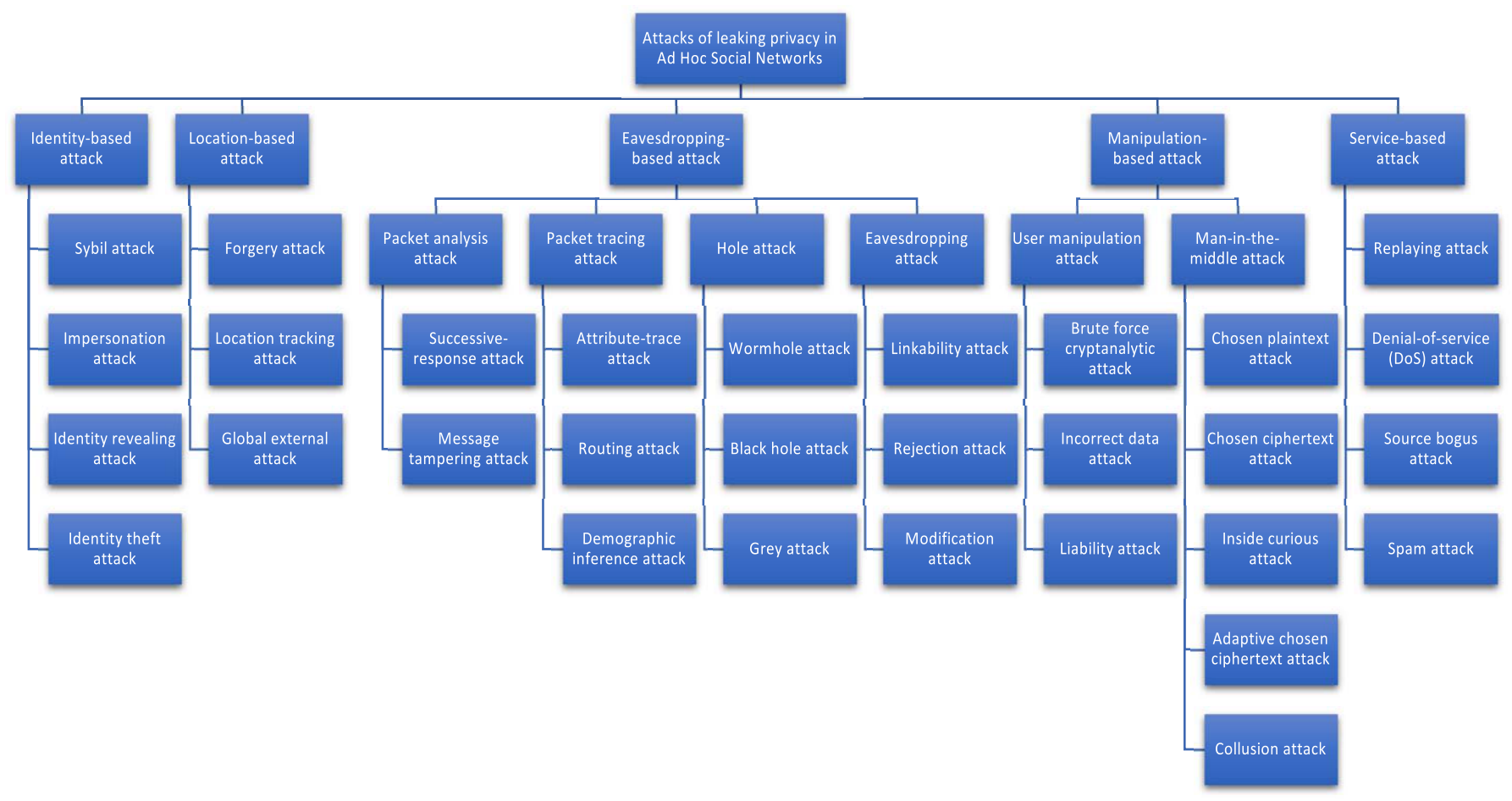

Fig. 3. Classification of attacks of leaking privacy in Ad Hoc Social Networks

- Forgery attack: In this attack, a forgery adversary generates a misleading message with the bogus location information in order to start the plotting attacks as the location tracking attack [31]. Using an explicit comparison-based approach, the PPM protocol in [37] has been proven with a theorem (non-forgeability) that any profile forgery attack can be detected. The SFPM protocol in [38] can resist to forgery attacks using a data processing center. The HealthShare scheme in [22] can be effectively resisted to the forgery attacks using the attribute-oriented authentication scheme. The PEC scheme in [33] can withstand to the forgery attacks via the group signature, which helps the trusted authority to track the user's unique identity. The PPBMA scheme in [11] can prevent the forgery attacks based on the verification of this equation, $M A C_{k_{j}^{i}}\left(M_{j}^{F} \| T_{j}^{F}\right)=? M A C_{j+1}^{F i}\left(M_{j}^{F} \| T_{j}^{F}\right)$.

- Global external attack: This attack is proposed in the paper [28], which can be classified in this category, i.e., locationbased attack. More precisely, a global external adversary tracks a vehicle in terms of Time, Location, and Velocity. The PCS strategy in [28] can resist to the global external attacks using two phases, including, 1) pseudonym self-delegated generation, and 2) conditional tracking.

\section{Eavesdropping-based attack}

This category of attacks is based on eavesdropping the network communications, and it consists of four major attacks; 1) eavesdropping attack, 2) packet analysis attack, 3) packet tracing attack, and 4) hole attack.

- Eavesdropping attack: When the nodes in ad hoc social network try to exchange the information on common interests, an eavesdropping attack tries to attain the transmitted data without the certificates. Then, it can perform some operations on this data using the linkability attack, rejection attack, and modification attack [38]. The HealthShare scheme in [22] is resistant to the eavesdropping attacks based on the ciphertext generated by delegated encryption algorithm. The PPBMA scheme in [11] is resistant against the eavesdropping attacks using the following condition: $M A C_{k_{j}^{i}}\left(M_{j} \| T_{j}\right) \neq M A C_{k_{j}^{i^{\prime}}}\left(M_{j} \| T_{j}\right)$. Based on semantic security, the PACP protocol in [10] has been proved that is semantically secure against the eavesdropping attacks. The SECSPP scheme in [9] has been proved that is secured against the eavesdropping attacks based on the authorization access phase.

- Packet analysis attack: This is a type of attack that is popular in wired networks, where an adversary captures the packets, and then it analyses in order to extract important information such as the common interests. In ad hoc social networks, this attack has the same strategic but the probability of launch is high compared to wired networks [24]. In addition, the successive-response attack [21] and the message tampering attack [38] could be run through the packet analysis attack. However, the SPRING protocol in [24] can resist to the packet analysis attacks using the anonymous authentication, which is based on a conditional privacy-preserving authentication technique. This technique is based on two key phases, including, 1) privacy-preserving authentication, and 2) conditional tracking. 
- Packet tracing attack: After eavesdropping the source and destination locations of packet, an adversary can trace this packet without need to recover the packet content [24]. In addition, when a security scheme uses the attributes, an attack can be launched, called the attribute-trace attack [22]. Therefore, the routing attack [40], [17] and demographic inference attack [32] could be run by tracing the packet controls used by the routing protocol. The SPRING protocol in [24] can resist to the packet tracing attacks based on the anonymous authentication.

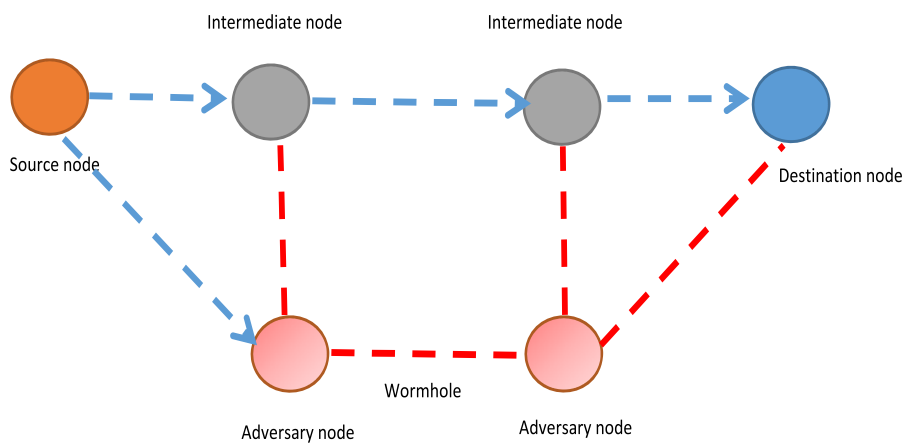

Fig. 4. Wormhole attack

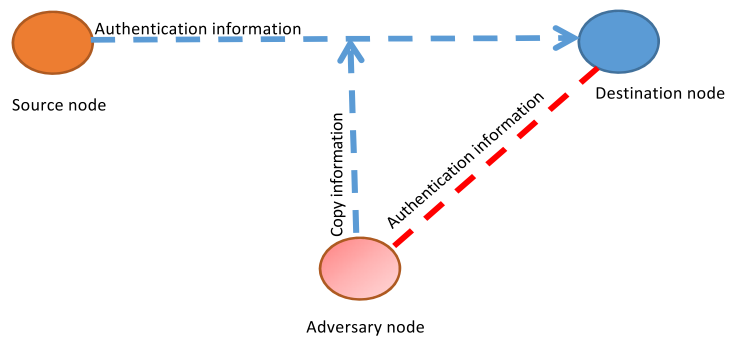

Fig. 5. Replay attack

TABLE VI

APPROACHES FOR DETECTING AND AVOIDING THE HOLE ATTACKS

\begin{tabular}{|l||l|l|l|}
\hline Scheme & Type & Data attacked & Approach \\
\hline $\begin{array}{l}\text { SPRING [24] } \\
(2010)\end{array}$ & Black/grey hole & Sensitive data & $\begin{array}{l}\text { Conditional authentication } \\
\text { technique }\end{array}$ \\
\hline $\begin{array}{l}\text { SDPP [17 } \\
(2014)\end{array}$ & Black hole & $\begin{array}{l}\text { Proactive Routing } \\
\text { data }\end{array}$ & $\begin{array}{l}\text { Cooperative neighbor X } \\
\text { neighbor }\end{array}$ \\
\hline $\begin{array}{l}\text { STAP [13] } \\
(2011)\end{array}$ & Black/grey hole & Sensitive data & Signature algorithm \\
\hline $\begin{array}{l}\text { ECPDR [40] } \\
(2013)\end{array}$ & Wormhole attack & User data & $\begin{array}{l}\text { Proxy re-signature cryp- } \\
\text { tography }\end{array}$ \\
\hline $\begin{array}{l}\text { SPF [18 } \\
(2010)\end{array}$ & Black/grey hole & Sensitive data & $\begin{array}{l}\text { Anonymous identity-based } \\
\text { encryption }\end{array}$ \\
\hline $\begin{array}{l}\text { EPSA [35] } \\
(2016)\end{array}$ & Wormhole attack & $\begin{array}{l}\text { Reactive Routing } \\
\text { data }\end{array}$ & $\begin{array}{l}\text { Cooperative neighbor X } \\
\text { neighbor }\end{array}$ \\
\hline
\end{tabular}

- Hole attack: This category consists of three types of attacks, including, wormhole attack, black hole attack, and grey hole attack. The hole attack is based on creating a communication tunnel where an adversary eavesdrops the communication inside the ad hoc social network through this tunnel, as shown in Fig. 4 Note that several adversaries can initiate the creation of the tunnel, where the routing protocol will be totally under the control of these adversaries. Tab VI. shows the approaches for detecting and avoiding the hole attacks in ad hoc social networks. For detecting blackhole attacks, the SDPP scheme proposed in [17] usse the cooperative neighbor technique and the homomorphic encryption method. The ECPDR scheme in [40] can resist to a wormhole attack using a restore strategy with the proxy re-signature cryptography technology. The EPSA scheme in [35] can detect and prevent a wormhole attack by using the cooperative neighbor $\mathrm{X}$ neighbor, its performance depending on the length of the tunnel created from the adversaries, i.e., the longer the tunnel, the higher the detection rate. Tracking the inside black/grey hole adversaries is possible with the STAP protocol which is presented in [13] with the use of the validity of $\operatorname{sig}(C T L)$, where sig is a signature algorithm and $C T L$ is the timestamp/location information. The SPF protocol [18] can resist to black/grey hole attacks using the anonymous identitybased encryption. The SPRING protocol [24] can also track inside black/grey hole adversaries by using the conditional 
TABLE VII

APPROACHES FOR DETECTING AND AVOIDING THE REPLAY ATTACKS

\begin{tabular}{|l||l|l|}
\hline Reference & Data attacked & Approach \\
\hline $\begin{array}{l}\text { Lu et al. [12] } \\
(2010)\end{array}$ & Inner data stored in the OBU & $\begin{array}{l}\text { Checks }\left|T^{\prime}-T\right| \leq \\
\Delta T\end{array}$ \\
\hline $\begin{array}{l}\text { Chim et al. [20] } \\
\text { (2014) }\end{array}$ & Local data of RSU & Timestamps \\
\hline $\begin{array}{l}\text { Xiong et al. [34] } \\
(2012)\end{array}$ & Data of member manager & Timestamps \\
\hline $\begin{array}{l}\text { Chim et al. [36] } \\
(2011)\end{array}$ & Data broadcast by the RSU & Timestamps \\
\hline $\begin{array}{l}\text { Huang et al. [10] } \\
(2011)\end{array}$ & All data in the network & Timestamps \\
\hline $\begin{array}{l}\text { Sun et al. [15] } \\
(2010)\end{array}$ & Data broadcast by the vehicles & Timestamps \\
\hline $\begin{array}{l}\text { Li et al. [9] } \\
(2008)\end{array}$ & Data transmitted between the vehicles & Sequence numbers \\
\hline $\begin{array}{l}\text { Yu et al. [29] } \\
\text { (2016) }\end{array}$ & Data broadcast by the vehicles & Timestamps \\
\hline
\end{tabular}

privacy-preserving authentication technique. In addition, the SPRING protocol can detect black/grey hole attacks with a detection algorithm, which is based on the distance $d\left(X_{i}\right)$ of each node $X_{i}$ in all vehicle nodes $V$ to the mean $\bar{X}$ and the thresholds $T_{B}, T_{G}$ for black hole attack and grey hole attack, where $d\left(X_{i}\right)=\left|X_{i}-\bar{X}\right|, \bar{X}=\frac{1}{|V|} \sum_{i=1}^{|V|} X_{i}$. The node is considered as a grey/black hole adversary when $d\left(X_{i}\right)>T_{\mathbf{G}}$ or $d\left(X_{i}\right)>T_{B}$.

\section{Manipulation-based attack}

This category of attacks is based on the manipulation of nodes of the Ad Hoc Social Network (users or socialspots), and it consists of two major attacks; 1) user manipulation attack and 2) man-in-the-middle attack.

- User manipulation attack: In this attack, an adversary tries to appear as a hotspot (small or large) for the nodes in ad hoc social network, in order to have the updates certificates. For example, an adversary sends a packet containing information of a false hotspot, then the node run the update certificates phase with this adversary. Therefore, these nodes have to honestly tell about their hotspots [19]. In addition, the brute force cryptanalytic attack, incorrect data attack, and liability attack [29], could be run through the user manipulation attack. The protocol proposed in [19] uses the authentication against the user manipulation attack. Specifically, this protocol is based on a privacy-preserving routing tree in order to make sensitive hotspots anonymous.

- Man-in-the-middle attack: The idea of this attack is like the idea in the hole attacks, but we classify it in this category because the adversary manipulates the users. The man-in-the-middle attack is especially applicable in the Diffie-Hellman key exchange method. As discussed in the survey [70], man-in-the-middle attack aims to compromise confidentiality, integrity, and availability. However, in order to reduce the security of the encryption scheme, the adversary can launch other types of attacks in this category as the chosen plaintext attack, chosen ciphertext attack, inside curious attack, and adaptive chosen ciphertext attack [39]. In addition, an adversary can launch a collusion attack [22], [33] where he tries to find two different packets $p 1$ and $p 2$ such that hash $(p 1)=\operatorname{hash}(p 2)$. The EP2DF scheme in [39] proposed a novel authentication framework, called lite-CA-based public key cryptosystem, to thwart the man-in-the-middle attacks and has been proved that is secure against adaptive chosen ciphertext attacks. Recall that the schemes resist against the eavesdropping attacks can resist against the collusion attacks. Based on the independent relation of the secret shares, the PEC scheme in [33] can avoid the collusion attacks.

\section{E. Service-based attack}

This category of attacks aiming to make a service unavailable of the network and it consists of four major attacks; 1) replaying attack, 2) denial-of-service (DoS) attack, 3) source bogus attack, and 4) spam attack.

- Replaying attack: When a node A wants to exchange data with node B, the node A must prove its identity, which the node B request a valid certificate, i.e., authentication information as shown in Fig. 5 . Then, the node A sent this certificate in a signed packet (SP). During this exchange, an adversary listening and saves this signed packet. Once the exchange is completed, this adversary tries to contact the node B. Hence, the node B request a valid certificate to adversary. The adversary sent the SP to the node B. At the end, the node B believes be dealing with the node A, and a service can be unavailable by the adversary. Note that this attack was discussed in several papers [12], [20], [34], [36], [10], [15], [9], [29]. Tab VII] shows the approaches for detecting and avoiding the replay attacks in ad hoc social networks. After the verification of the identity $P I D i$, by a valid time interval $\Delta T$ for transmission delay, the intelligent parking scheme in [12] can avoid the replaying attacks when the RSU checks the following condition : $\left|T^{\prime}-T\right| \leq \Delta T$. Similarly to 
the scheme in [12], the scheme in [20], the scheme in [34], the PASS scheme in [15], and the SECSPP scheme in [9] checks the timestamps in the messages to reduce the impact of replay attack. When the RSU stores the pseudo-identities used by vehicles, the SPECS scheme in [36] can avoid the replay attacks with the help of RSU, which he can checks the pseudo-identity in its database. The PACP protocol in [10] can avoid the replay attacks with the use of authentication and sequence numbers. The MixGroup scheme in [29] can avoid the replay attacks with the use of timestamps in the revocation operation.

- Denial-of-service (DoS) attack: This attack is the heart of this category. During the social communications, an adversary can launch a DoS attack in order to put a service unavailable, for example, disruption of routing process, block a file server, wasting the limited buffer resource, or preventing the distribution of secret keys. Therefore, DoS attack can be launched from several layers, i.e., link layer, physical layer, network layer, transport layers, and application layers [65]. To detect the Denial-of-service (DoS) attacks, the PASS scheme in [15] adopt the Schnorr signature algorithm and the prestore strategy to signing certificate $\operatorname{Cert}_{T A, V_{i}, k}$ and pseudonymous certificate $\operatorname{Cert}_{T A, V_{i}, j}$.

- Source bogus attack: This attack is qualified as an inside attack, which a source deliberately inject bogus data in the social communications to wasting the limited buffer resource of the nodes [13]. In addition, this attack is similar to the incorrect data attack [29] but with other objectives. Note that we found only Lin et al. [13] that discuss the source bogus attack in the ad hoc social networks until now. The STAP protocol in [13] uses the single-attribute encryption against the source bogus attacks.

- Spam attack: This attack is very popular in electronic mails. In general, an adversary tries to send several e-mails to multiple receivers whose addresses have generally been recovered on the internet. The first goal of this attack is to do advertising at lower prices. However, an adversary can launch this attack in ad hoc social networks in order firstly to disrupt the data filtering and secondly to spy the storage space. Note that we found only Hameed et al. [30] that discuss the spam attack in the ad hoc social networks until now. The LENS system in [30] can prevent the spam transmission using the idea of Gate keepers.

TABLE VIII

CRYPTOGRAPHIC METHODS USED IN PRIVACY-PRESERVING SCHEMES FOR MSNS

\begin{tabular}{|c|c|c|c|c|c|c|c|c|c|c|c|c|}
\hline & \multicolumn{12}{|c|}{ Privacy-preserving schemes } \\
\hline Cryptographic methods & [37] & [38] & 40 & [14 & {$[17$} & [35] & $\llbracket 19$ & [22] & [27] & [33] & [23] & [41] \\
\hline Secure cryptographic hash functions $[71]$ & & & $\checkmark$ & $\checkmark$ & $\checkmark$ & $\checkmark$ & $\checkmark$ & $\checkmark$ & $\checkmark$ & $\checkmark$ & $\checkmark$ & \\
\hline Homomorphic encryption [72] & $\checkmark$ & & & & $\checkmark$ & & & & & & & $\checkmark$ \\
\hline Multiple pseudonym technique [73] & & & & & & & $\checkmark$ & & & & & \\
\hline HMAC [74] & & $\checkmark$ & & & & & & & & & & \\
\hline Short signatures technique $[75$ & & & $\checkmark$ & & & $\checkmark$ & & & & & & \\
\hline Identity-Based Aggregate Signatures [76] & & & & $\checkmark$ & & & & & & & & \\
\hline Identity-based encryption [77] & & & & & $\checkmark$ & & & & & & & \\
\hline PKE with keyword search [78] [79] & & & & & & $\checkmark$ & & & & & & \\
\hline Linear Secret Sharing $[80]$ & & & & & & & & $\checkmark$ & & $\checkmark$ & & \\
\hline Attribute-based encryption [81] & & & & & & & & & & $\checkmark$ & & \\
\hline Hidden vector encryption [82] 83 & & & & & & & & & & & $\checkmark$ & \\
\hline Short group signature [51] & & & & & & & & & & $\checkmark$ & & \\
\hline
\end{tabular}

\section{COUnTERMEASuRES AND GAME THEORETIC APPROACHES}

Most privacy preserving schemes for ad hoc social networks that we have examined use the cryptography as a countermeasure in order to preserve privacy. Generally, cryptography is one of the disciplines of cryptology, which was initialy proposed in order to protect data, i.e., ensuring confidentiality, authenticity, and integrity using secrets or keys. Fig. 6 presents the taxonomy of cryptographic primitives [94]. The cryptographic methods used in privacy-preserving schemes for MSNs and VSNs are summarized in Tab. VIII and Tab. IX] respectively. In order to prove these security schemes theoretically, researchers can use game theoretic approaches [95], [96]. In this section, we will discuss the state of the art of privacy-preserving schemes for VSNs and MSNs and we provide a description and types of game theoretic models for preserving privacy in ad hoc social networks.

\section{A. Symmetric-key primitives}

Symmetric-key primitives can be classified on three types of techniques, including, symmetric key-ciphers, keyed hash function, and pseudo-random sequences. In order to secure data at the MAC layer, AES block cipher algorithm was proposed in the IEEE 802.15.4 [97]. Yang et al. [38] used keyed-hashing for message authentication code (HMAC) [74] in order to achieve the integrity of the message and source data authentication. To compute HMAC, the scheme [38] chooses a secret key for users $K_{j}\left(\left|K_{j}\right|=128\right)$ for $U_{j} \in \mathrm{U}$ and $K_{A}\left(\left|K_{A}\right|=128\right)$ for $U_{A}$, then it sends $\left(s, K_{j}\right)$ and $\left(d, K_{A}\right)$ to $U_{j}$ and $U_{A}$, respectively, where $s, d \in \mathrm{Z}_{p}$. 
TABLE IX

CRYPTOGRAPHIC METHODS USED IN PRIVACY-PRESERVING SCHEMES FOR VSNS

\begin{tabular}{|c|c|c|c|c|c|c|c|c|c|c|c|c|c|c|c|c|c|}
\hline \multirow[b]{2}{*}{ Cryptographic methods } & \multicolumn{17}{|c|}{ Privacy-preserving schemes } \\
\hline & 25 & 28 & [12 & 20 & [31 & 34 & 111 & [36] & [39] & [10 & [15 & [13] & [18] & 21 & 24 & 9 & 29 \\
\hline Secure cryptographic hash functions & $\checkmark$ & $\checkmark$ & & $\checkmark$ & & $\checkmark$ & $\checkmark$ & $\checkmark$ & $\checkmark$ & $\checkmark$ & $\checkmark$ & $\checkmark$ & $\checkmark$ & $\checkmark$ & $\checkmark$ & $\checkmark$ & $\checkmark$ \\
\hline Verifier-local revocation $[84]$ & $\checkmark$ & & & & & & & & & & & & & & & & $\checkmark$ \\
\hline Short signatures technique & & $\checkmark$ & & $\checkmark$ & & & & $\checkmark$ & & $\checkmark$ & & & & & & & $\checkmark$ \\
\hline Ephemeral key $[85]$ & & & $\checkmark$ & & & & & & & & & & & & & & \\
\hline Identity-based encryption & & & $\checkmark$ & & & & & & & $\checkmark$ & & & & & & & \\
\hline Proxy Re-encryption $[86$ & & & & $\checkmark$ & & & & & & & & & & & & & \\
\hline ID-based signature $[87]$ & & & & & $\checkmark$ & & & & & & & & & & & & \\
\hline ID-based online/offline signature [88] & & & & & $\checkmark$ & & & & & & & & & & & & \\
\hline Ring signature $[59]$ & & & & & & $\checkmark$ & & & & & & & & & & & \\
\hline Certificateless public key [89][90] & & & & & & & & & $\checkmark$ & & & & & & & & \\
\hline Schnorr signature algorithm 91$]$ & & & & & & & & & & & $\checkmark$ & & & & & & \\
\hline Elliptic curve (ECDSA) & & & & & & & & & & & & & & $\mathrm{v}$ & & & \\
\hline CPPA Technique $[60]$ & & & & & & & & & & & & & & & $\checkmark$ & & \\
\hline Blind signature $[62] 63$ & & & & & & & & & & & & & & & & $\checkmark$ & \\
\hline Multiple pseudonym technique [73] & & & & & & & & & & & & & & & & & $\checkmark$ \\
\hline
\end{tabular}

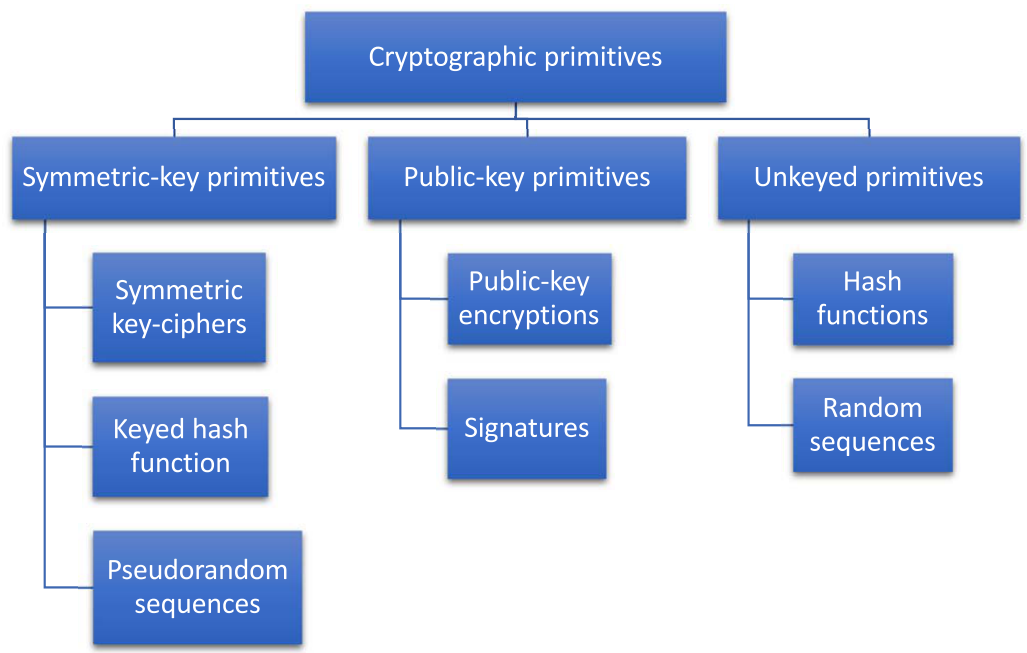

Fig. 6. Taxonomy of cryptographic primitives

\section{B. Public-key primitives}

Public-key primitives can be classified on two types of techniques, including, public-key encryptions and signatures. We note that the public-key primitives is used mostly by privacy-preserving schemes.

Public-key encryption is potentially a good security solution when the number of nodes is very high. The schemes [17], [12], [10] use the identity-based encryption [77]. When an OBU with identifier $I D_{i}$ registers itself to the system, the proposed scheme uses the secret key $s$ to encrypt the real identifier $I D_{i}$ into a pseudo-ID $P I D_{i}=\operatorname{Encs}_{s}\left(I D_{i} \| r_{i}\right)$, where $r_{i}$ is randomly chosen from $\mathrm{Z}_{q}^{*}$. Then, the OBU encrypts the message $M$ based on pseudo-ID $P I D_{i}$, the current timestamp $T$, and the ephemeral key. The scheme [35] uses the public key encryption with keyword search [78], [79], which is takes as input the public key $P K$ and keywords $\left(w_{1}, w_{2}, \cdots, w_{l}\right)$ that is associated with one document in order to output a ciphertext $C$. Then, it uses the trapdoor information $T_{w_{1}^{\prime}{ }_{1}, w^{\prime}{ }_{2}, \cdots, w^{\prime} l}$ and $\operatorname{Test}\left(C, T_{w_{1}^{\prime}, w^{\prime}{ }_{2}, \cdots, w^{\prime} l}\right)$.

Based on user attributes, Liang et al. [33] adopted the attribute-based encryption [81], which outputs a ciphertext associated with the attribute set. Following a different approach and focusing on achieving efficient fine-grained filtering, Zhang et al. [23] used the hidden vector encryption [82], [83]. Specifically, the filter creator in the scheme [23] generates his fine-grained keyword filter as a vector $w=\left(w_{1}, \cdots, w_{l}\right) \in\{1, \cdots, n\}^{l}$.

Dong et al. [39] proposed the certificate less public key (CL-PKC) [89], [90] in order to achieve lightweight public key certificate management. The CL-PKC is specified by the following seven randomized algorithms: Setup, Partial-PrivateKey-Extract, Set-Secret-Value, Set-Private-Key, Set-Public-Key, Encrypt, and Decrypt. The Setup algorithm is run by the key generating center (KGC), which outputs the system parameters params and master - key. The Partial-Private-Key-Extract algorithm returns a partial private key $D_{A}$ using params, master - key, and an identifier for entity $A, I D_{A} \in\{0,1\}^{*}$. The Set-Secret-Value algorithm outputs $A$ 's secret value $x_{A}$ using params and $I D_{A}$. The Set-Private-Key algorithm outputs the 
(full) private key $S_{A}$ using $I D_{A}$, and $x_{A}$. The Set-Public-Key algorithm constructs the public key $P_{A}$ for entity $A$ using params and $x_{A}$. The Encrypt algorithm outputs a ciphertext $C$ using $P_{A}$ and $I D_{A}$. The Decrypt algorithm returns a message $M$ using params, $C$, and $S_{A}$. Chim et al. [20] proposed a proxy re-encryption [86] that is based on the following algorithm:

TABLE $X$

GAME THEORETIC APPROACHES USED IN PRIVACY-PRESERVING SCHEMES

\begin{tabular}{|l||l|l|}
\hline Scheme & Approach & Main results \\
\hline $\begin{array}{l}\text { ECPDR scheme [40] } \\
(2013)\end{array}$ & Static game & Protect location privacy \\
\hline $\begin{array}{l}\text { Scheme [19] } \\
(2012)\end{array}$ & Cooperation game & $\begin{array}{l}\text { Determine the optimal data forward- } \\
\text { ing strategy }\end{array}$ \\
\hline $\begin{array}{l}\text { SSH scheme [27] } \\
(2011)\end{array}$ & Sequence games & $\begin{array}{l}\text { Prove that } I B E \text { is IND-sPS-CPA se- } \\
\text { cure under the k-SPDBH assump- } \\
\text { tion in the random oracle model }\end{array}$ \\
\hline $\begin{array}{l}\text { PCS strategy [28] } \\
(2012)\end{array}$ & $\begin{array}{l}\text { Noncooperative informa- } \\
\text { tion static game }\end{array}$ & $\begin{array}{l}\text { Prove that the feasibility of the } \\
\text { pseudonym changing at social spots } \\
\text { (PCS) strategy }\end{array}$ \\
\hline $\begin{array}{l}\text { SPECS scheme [36] } \\
(2011)\end{array}$ & Static game & $\begin{array}{l}\text { Protect message integrity and authen- } \\
\text { tication }\end{array}$ \\
\hline $\begin{array}{l}\text { FLIP protocol [21] } \\
(2010)\end{array}$ & Sequence games & $\begin{array}{l}\text { Demonstrate that the protocol is se- } \\
\text { cure in the VANET scenarios }\end{array}$ \\
\hline $\begin{array}{l}\text { The scheme [84] } \\
(2004)\end{array}$ & Traceability game & $\begin{array}{l}\text { Prove that the group signature scheme } \\
\text { satisfies the requirements traceability }\end{array}$ \\
\hline
\end{tabular}

Setup, KeyGen, Encrypt, Decrypt, RKGen, Reencrypt. The Setup algorithm is used in order to output both the master public parameters and the master secret key. The KeyGen algorithm outputs a decryption key $s k_{i d}$ corresponding to identity $i d$. The Encrypt algorithm outputs $c_{i d}$, the encryption of message under the specified identity. The Decryp talgorithm decrypts the ciphertext $c_{i d}$ using the secret key $s k_{i d}$. The RKGen algorithm produces a re-encryption key $r k_{i d_{1} \rightarrow i d_{2}}$. The Reencrypt algorithm outputs a "re-encrypted" ciphertext $c_{i d_{2}}$.

Li et al. [9] presented the non-interactive ID-based public-key cryptography [93], which is based on three following phases, namely: system setup, user registration, and authentication, respectively. The system setup phase outputs the public key $e$ in $Z_{\emptyset(N)}^{*}$ and a corresponding private key $d$ where $e * d \equiv 1(\bmod \emptyset(N))$. The user registration phase outputs a secret key $s_{i}=e * \log _{g}\left(I D_{i}^{2}\right)(\bmod \emptyset(N))$ for the node $U_{i}$. The authentication phase verifies $Y=\left(I D_{i}^{2}\right)^{r * s_{j}}=\left(I D_{i}^{2}\right)^{r * s_{j}}(\bmod \emptyset(N))$.

Homomorphic encryption [72] is used by three schemes [37], [17] [41]. Rothblum in [98] considers homomorphic encryption as a public-key scheme. Specifically, homomorphic encryption uses the public key $(N, g)$ and the corresponding private key $\operatorname{sk}(\lambda, \delta)$ in order to construct both the ciphertext $c$ and the plaintext $m$. The ciphertext $c=g^{m} \cdot r^{n} \bmod N$ where $r$ is a random number. The plaintext $m=L\left(c^{\lambda \bmod N^{2}}\right) \cdot \delta \bmod N$, where $(x)=(x-1) / N$. The additive homomorphic property is as follows: $\left(m_{1}\right) \cdot E\left(m_{2}\right)=\left(g^{m_{1}} \cdot r_{1}^{n}\right)\left(g^{m_{2}} \cdot r_{2}{ }^{n}\right) \bmod N^{2}=E\left(m_{1}+m_{2}\right)$.

To achieve anonymous authentication, the schemes in [28], [20], [36], [10], [29] [40], [35] use the Boneh-Boyen short signature [75]. In general, the following three algorithms specify a signature scheme: KeyGen, Sign, and Verify. The KeyGen algorithm outputs a random key pair $(P K, S K)$. The Sign algorithm constructs a signature $\sigma$ using a private key $S K$ and a message $M$. The Verify algorithm verifies the signature and returns valid or invalid. The Boneh-Boyen short signature [75] is based on these three algorithms: Key Generation, Signing, and Verification. The Key Generation algorithm is same as KeyGen. The Signing algorithm outpust the signature $(b, \operatorname{Sign}(m))$ where $\sigma=H_{1}(S K, M) \in\{0,1\}, m=H_{2}(b, M), H_{1}$ and $H_{2}$ two hash functions. The Verification algorithm outputs valid if Verify $\left(P K, H_{2}(b, M), \sigma\right)=$ valid. Note that there is the ID-based signature [87] and the ID-based online/offline signature [88] which are used by the scheme in [31].

Liang et al. in [33] proposes a short group signature [51] that is based on the following algorithm: Setup, Join, Sign, Verify, and Trace, which could be executed by three parties: group manager, user, and verifier. The Setup algorithm outputs the public parameters $P P$, the master key $M K$, and the tracing key $T K$, where $P P=(g, h, Z) \in G \times G_{q} \times G_{p}, M K=z \in \mathrm{Z}_{n}^{*}$, $T K=q \in$ Z. $n=p q$ where $p, q$ are random primes and $G$ is a cyclic bilinear group and its subgroup $G_{p}$ and $G_{q}$ of respective order $p$ and $q . g$ is a generator of $G$ and $h$ is a generator of $G_{q}$. The Join algorithm construct the secret key $K_{i d}=\left(s_{i d}, g^{\frac{1}{z+s_{i d}}}\right)$ , where $s_{i d}$ is a user's identity $i d$. The Sign algorithm outputs signature $\sigma=\left(\sigma_{1}, \sigma_{2}, \sigma_{3}, \pi_{1}, \pi_{2}\right) \in G^{5}$. The Verify algorithm verifies $T_{1}=? e\left(h, \pi_{1}\right)$ and $T_{2}=? e\left(h, \pi_{2}\right)$. The Trace algorithm trace the identity of signer using $P P, T K$, and $\sigma$. Moreover, note that group signatures with verifier-local revocation [84] are used in both the scheme in [25] and the scheme in [29]. In addition, a blind signature [62], [63] is used in the scheme [9], a schnorr signature algorithm [91] is used in the scheme [15], and a ring signature [59] is used in the scheme [34].

\section{Unkeyed primitives}

Unkeyed primitives can be classified on two types of techniques, including, hash functions and random sequences. The secure cryptographic hash functions [71] are used in most privacy-preserving schemes for MSNs and VSNs, where the cryptographic 
hash function is used in order to check the integrity of a message. For example, modifying a message when transmitting can be proved by comparing the message hash value before and after transmission. Specifically, using a security parameter $\lambda$, a hashing function $\mathcal{H}:\{0,1\}^{*} \rightarrow\{0,1\}^{\lambda}$ is cryptographically secure if it satisfies three security properties, namely, pre-image-resistance, second pre-image resistance, and collision-resistance.

\section{Game theoretic approaches}

To prove the feasibility of privacy-preserving schemes in practice, researchers in the security field use various mathematical tools such as game theoretic approaches [95]. Since the efficiency of cryptographic methods has already been proved, we note that only a small fraction of privacy-preserving schemes that we have examined, used game theoretic approaches, which are summarized in Tab. X] More precisely, Ferrag et al. in [40] use the static game [99] in the ECPDR scheme to protect location privacy. Liang et al. in [19] uses the cooperation game to determine the optimal data forwarding strategy. The SSH scheme [27] uses the sequence games to prove that $I B E$ is IND-sPS-CPA is secure under the k-SPDBDH assumption in the random oracle model. The pseudonym changing at social spots (PCS) strategy [28] uses non-cooperative information static game [99] to prove the feasibility of PCS. Similarly to the scheme [40], Chim et al. in [36] use the static game to protect message integrity and authentication. The sequence games is used by the FLIP protocol [21] to demonstrate that the protocol is secure in the VANET scenarios. Finally, the scheme [84] use the traceability game to prove that the group signature scheme satisfies the requirements traceability. For more details, we refer the reader to the survey [95].

TABLE XI. Summary of privacy-preserving schemes for MSNs (Published between 2011 and 2016)

\begin{tabular}{|c|c|c|c|c|c|}
\hline Scheme & $\begin{array}{l}\text { Network } \\
\text { model }\end{array}$ & Privacy model & Goals & Main phases & $\begin{array}{l}\text { Performances (+) and lim- } \\
\text { itations (-) }\end{array}$ \\
\hline $\begin{array}{l}\text { Liang } \\
\text { et al. } \\
\text { [37 } \\
(2013)\end{array}$ & $\begin{array}{l}\text { Each user has a } \\
\text { profile with di- } \\
\text { mension vector } \\
\text { to find the tar- } \\
\text { geting user }\end{array}$ & $\begin{array}{l}\text { Non-anonymity; } \\
\text { Conditional } \\
\text { anonymity; } \\
\text { Full Anonymity }\end{array}$ & $\begin{array}{l}\text { Users compare their } \\
\text { profiles while not } \\
\text { disclosing the pro- } \\
\text { files }\end{array}$ & $\begin{array}{l}\text { Explicit comparison-based } \\
\text { approach; } \\
\text { Implicit comparison-based } \\
\text { approach; } \\
\text { Implicit predicate-based ap- } \\
\text { proach }\end{array}$ & $\begin{array}{l}\text { + Anonymity break period } \\
+ \text { Anonymity risk level } \\
\text {-Limited analysison the } \\
\text { impact of "=" on the } \\
\text { anonymity }\end{array}$ \\
\hline $\begin{array}{l}\text { Yang et } \\
\text { al. |38| } \\
(2016)\end{array}$ & $\begin{array}{l}\text { Specified by } \\
\text { a trusted key } \\
\text { distribution } \\
\text { center with a } \\
\text { semi-trusted } \\
\text { data processing } \\
\text { center }\end{array}$ & $\begin{array}{l}\text { Privacy } \\
\text { preservation the } \\
\text { profiles of users }\end{array}$ & $\begin{array}{l}\text { Minimize the per- } \\
\text { sonal profiles dis- } \\
\text { closure }\end{array}$ & $\begin{array}{l}\text { Cosine similarity matching; } \\
\text { Weighted } l_{1} \text {-norm matching }\end{array}$ & $\begin{array}{l}\text { + Computation complexity } \\
\text { + Average running time vs. } \\
\text { number of profile items } \\
\text {-The layer routing is not } \\
\text { considered }\end{array}$ \\
\hline $\begin{array}{l}\text { Ferrag } \\
\text { et al. } \\
{[40]} \\
(2013)\end{array}$ & $\begin{array}{l}\text { Each user has } \\
\text { a sociality } \\
\text { strength in a } \\
\text { set of social } \\
\text { hotspots }\end{array}$ & $\begin{array}{l}\text { Immutability; } \\
\text { Transparency; } \\
\text { Accountability; }\end{array}$ & $\begin{array}{ll}\text { Improve routing } \\
\text { by } & \text { privacy } \\
\text { preservation } & \end{array}$ & $\begin{array}{l}\text { Node certificate updating; } \\
\text { Message signature and ver- } \\
\text { ification; } \\
\text { Response Requested; } \\
\text { Demand Response }\end{array}$ & $\begin{array}{l}\text { + Black hole detection rate } \\
+ \text { Transmission delay } \\
\text {-Limited analysis with few } \\
\text { adversaries }\end{array}$ \\
\hline $\begin{array}{l}\text { Liang } \\
\text { et al. } \\
{[14 \mid} \\
(2014)\end{array}$ & $\begin{array}{l}\text { Multiple } \\
\text { vendors } \\
\text { offering similar } \\
\text { services to } \\
\text { users where } \\
\text { each vendor } \\
\text { is equipped } \\
\text { with a wireless } \\
\text { communication } \\
\text { device }\end{array}$ & Trust evaluation & $\begin{array}{l}\text { Detect and prevent } \\
\text { the sybil attacks }\end{array}$ & $\begin{array}{l}\text { Structured reviews; } \\
\text { Synchronization tokens; } \\
\text { Review generation and sub- } \\
\text { mission }\end{array}$ & $\begin{array}{l}\text { + Provides a good security } \\
\text { analysis of sybil attacks } \\
\text { - No comparison with other } \\
\text { systems }\end{array}$ \\
\hline $\begin{array}{l}\text { Ferrag } \\
\text { et al. } \\
{[17 \mid} \\
(2014)\end{array}$ & $\begin{array}{l}\text { Peer-to- } \\
\text { peer node } \\
\text { community } \\
\text { with a large } \\
\text { number of } \\
\text { mobile users }\end{array}$ & $\begin{array}{l}\text { Transparency; } \\
\text { Impersonator resis- } \\
\text { tance }\end{array}$ & $\begin{array}{l}\text { Provides the } \\
\text { strong privacy- } \\
\text { preservation of } \\
\text { message; } \\
\text { Provides the } \\
\text { evolution of users' } \\
\text { certificates }\end{array}$ & $\begin{array}{l}\text { Detecting attacks; } \\
\text { Response Requested; } \\
\text { Demand Response; } \\
\text { Certificate evolution }\end{array}$ & $\begin{array}{l}\text { + Detectreq reporting delay } \\
+ \text { Transmission delay } \\
\text { - Need of large Detectreq } \\
\text { reporting }\end{array}$ \\
\hline
\end{tabular}




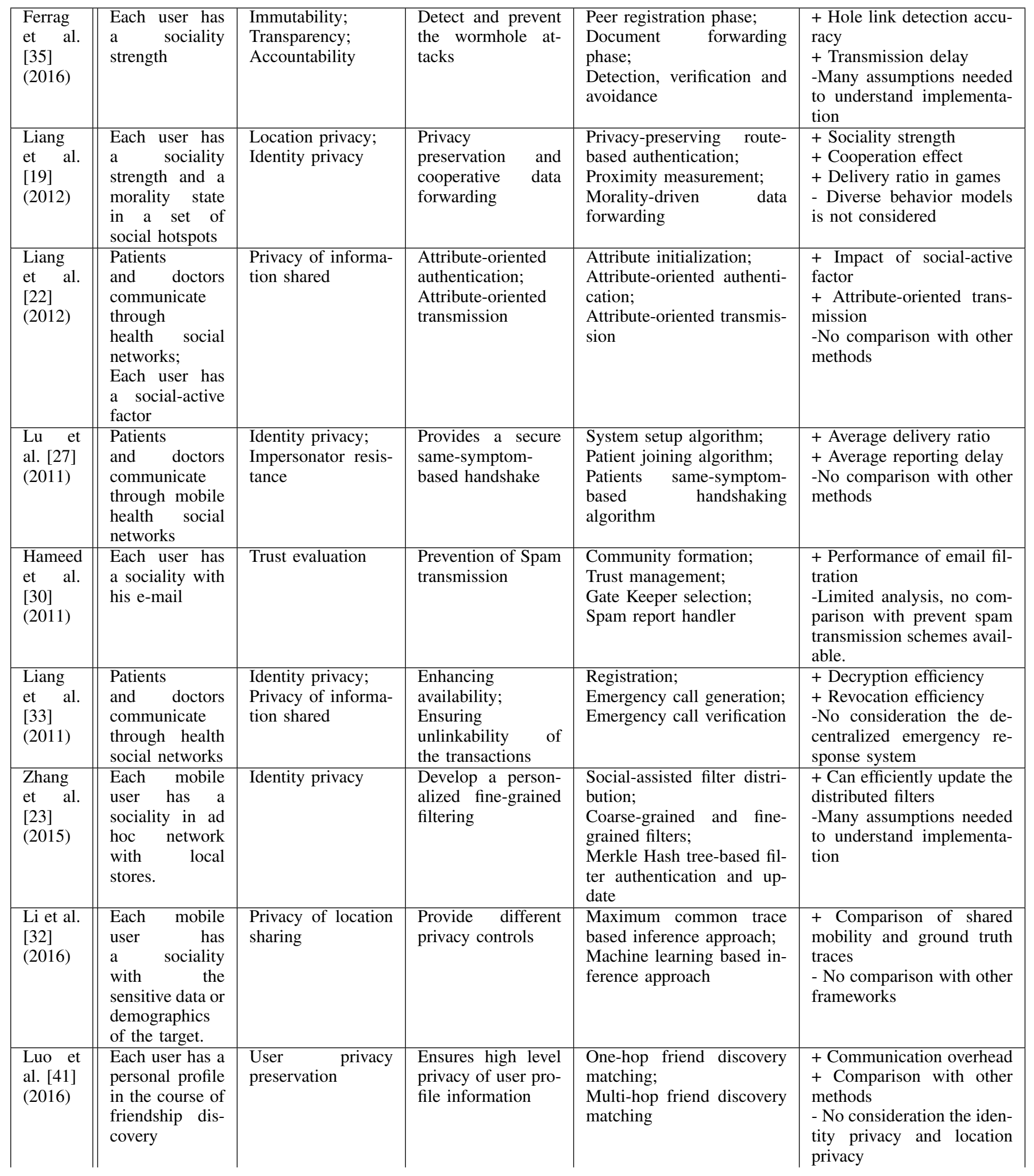




\section{PRIVACY-PRESERVING SCHEMES FOR MSNS}

In this section, we in-detail examine fourteen privacy-preserving schemes developed for or applied in the context of MSNs. Based on the network model, we classify these schemes in six categories, including, social profile, social morality, social routing, social health, location-based services, and service-oriented sociality. In addition, these schemes as shown in Tab. XI are published between 2011 and 2016.

\section{A. Network model with social profile}

In [37], Liang et al. considers that each user has a profile represented by a distinct dimension vector that can be used to find the targeting user. Specifically, the work in [37] presents a scheme, called PPM, which can preserve three privacy models, including, 1) non-anonymity, 2) conditional anonymity, and 3) full anonymity. With the PPM scheme, users can compare their profiles while not disclosing the profiles. The PPA uses three main phases, namely, explicit comparison, implicit comparison, and implicit predicate. The PPM scheme is efficient in terms of anonymity break period and anonymity risk level, but the article fails to provide a detailed analysis on the impact of "=" on the anonymity. Privacy preserving of the profiles of users is an important topic as identified in [38]. Yang et al. in [38] characterized a MSN by a trusted key distribution center with a semi-trusted data processing center and he proposed a privacy preserving protocol called SFPM. In order to minimize the personal profiles disclosure, the SFPM protocol uses two main phases of matching, including, 1) cosine similarity and 2) weighted $l_{1}$-norm. The SFPM protocol is efficient in terms of computation complexity and average running time vs. number of profile items, but the layer routing is not considered. In another recent work [41] Luo et al. proposes a privacy-preserving multi-hop profile-matching protocol for proximity-based mobile social networks.

\section{B. Network model with social morality}

In [19], authors state that each user has a sociality strength and a morality state in a set of social hotspots. Specifically, Liang et al. [19] developed a protocol for privacy preservation and cooperative data forwarding, which can protect both the location privacy and the identity privacy of the user. This protocol uses three main phases, including, 1) privacy-preserving route-based authentication, 2) proximity measurement, and 3) morality-driven data forwarding. In addition, this protocol is efficient in terms of sociality strength, cooperation effect, and delivery ratio in games, but diverse behavior models are not considered.

\section{Network model with social routing}

The routing protocol in social ad hoc networks is a principal element to efficiently route the produced social data. The works in [40], [17], [35] consider the sociality in routing protocols as the OLSR protocol [100] and the AODV protocol [101]. Ferrag et al. in [40] developed a scheme, called ECPDR, in order to improve routing by incorporating the privacy preservation dimension. The ECPDR scheme can provide immutability, transparency, and accountability. For detecting attacks, the ECPDR scheme uses four main phases, including,1) node certificate updating, 2) message signature and verification, 3) response requested, and 4) demand response. In addition, the ECPDR scheme is efficient in terms of black hole detection rate and transmission delay, but gives a limited analysis with few adversaries. Ferrag et al. [17] considering a peer-to-peer node community with a large number of mobile users proposes a novel scheme, called SDPP. Based on the certificate evolution phase, the SDPP scheme can provide transparency and impersonator resistance. The SDPP scheme is efficient in terms of reporting delay and transmission delay, but leads to the creation of large reports. Ferrag et al. [35] focuses on detecting and preventing the wormhole attacks and proposes a scheme called EPSA. The EPSA scheme is based on three main phases, including, 1) peer registration, 2) document forwarding, and 3) detection, verification and avoidance. The ESPA scheme is efficient in terms of hole link detection accuracy and transmission delay, but makes too many assumptions regarding the network characteristics.

\section{Network model with social health}

The health social networks (HSNs) which are essential for the communication between patients and doctors demand highly efficient privacy-preserving schemes. In [22], Liang et al. considers that each user has a social-active factor in HSN. The HealthShare scheme in [22] is proposed in order to provides privacy of information shared, which is devided in three main phases including, 1) attribute initialization, 2) attribute-oriented authentication, and 3) attribute-oriented transmission. The HealthShare scheme is efficient in terms of the impact of social-active factor and the attribute-oriented transmission, but the article doesn't present a comparison of the proposed mechainsm with other methods. In [33], Liang et al. developed a scheme, called PEC, for HSN. The PEC scheme ensures unlinkability of the transactions and it is based on two main algorithms; 1) emergency call generation and 2) emergency call verification.The PEC scheme is efficient in decryption and revocation, but needs the consideration of the decentralized emergency response system. Similarly to [22] and [33], in [27], Lu et al. proposed a 
scheme, called SSH, which targets mobile users in HSNs. The SSH scheme provides a secure same-symptom-based handshake based on two main phases, including,1) patient joining and 2) patients same-symptom-based handshaking. In addition, the SSH scheme is efficient in delivery ratio and reporting delay, but authors dont present a thorough comparison of their system with othersimilar methods.

\section{E. Network model with location-based services}

The geosocial networking is a new concept in the topic of social networking, where each mobile user has a sociality whcih is associated with some sensitive data or the demographics of the target. In [32], an interesting recent work considers the demographics (e.g., age, gender, education) in MSN, and based on these the authors proposed a new set of attacks that can infer the demographics. Specifically, in order to provide full privacy of location sharing, one needs to combine the following approaches in [32]: 1) maximum common trace based inference approach and 2) machine learning based inference approach. The work in [32] presents a good comparison of shared mobility and ground truth traces. In addition, the work in [32] developed a framework, called SmartMask, for the protection of location privacy, which needs to be compared with other frameworks in the future in order to test its efficiency. The idea of social spot in MSN proposed in the VSLP protocol [102] can be applied in this category.

\section{F. Network model with service-oriented sociality}

Liang et al. [14] developed a system, called TSE, which considers multiple vendors offering similar services to users where each vendor is equipped with a wireless communication device. The TSE system provides a trust evaluation mechanism and can detect and prevent a sybil attacks. The TSE system is based on three main phases, namely, structured reviews, synchronization tokens, and review generation and submission. The work in [14] provides a good security analysis of the mechanism against sybil attacks, but lacks comparison with other systems. Similarly to the TSE scheme [14], the LENS scheme [30] also provides a trust evaluation and prevention mechanism against spam transmission. Zhang et al. [23] developed a personalized fine-grained filtering scheme, called PIF. The PIF scheme considers that each mobile user has a sociality in an ad hoc network with local stores. The PIF scheme is based on three main phases, namely, social-assisted filter distribution, coarse-grained and finegrained filters, and merkle hash tree-based filter authentication and update. In addition, the PIF scheme can efficiently update the distributed filters but makes too many assumptions regarding the network characteristics. 
TABLE XII. Summary of privacy-preserving schemes for VSNs (Published between 2008 and 2016)

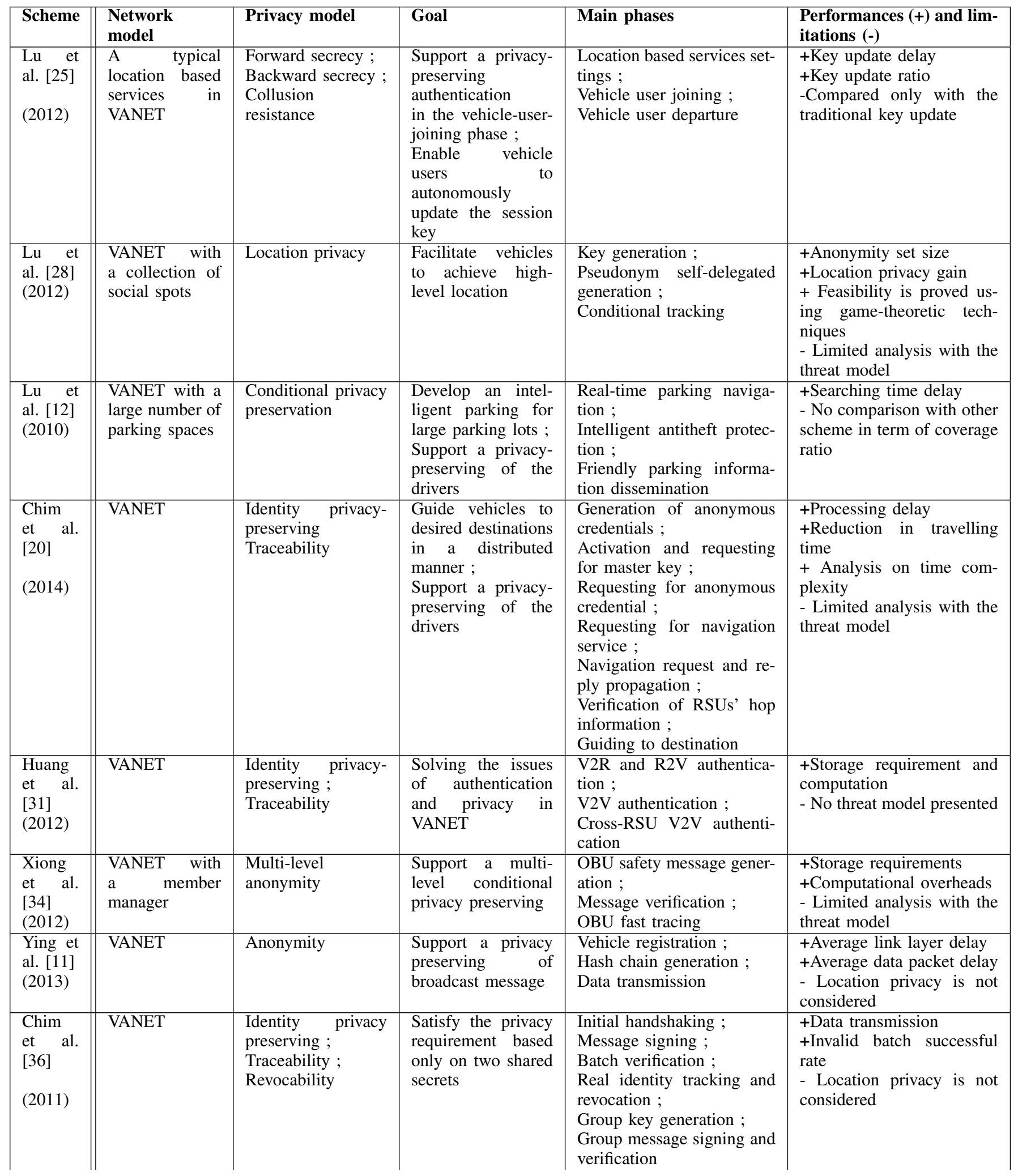




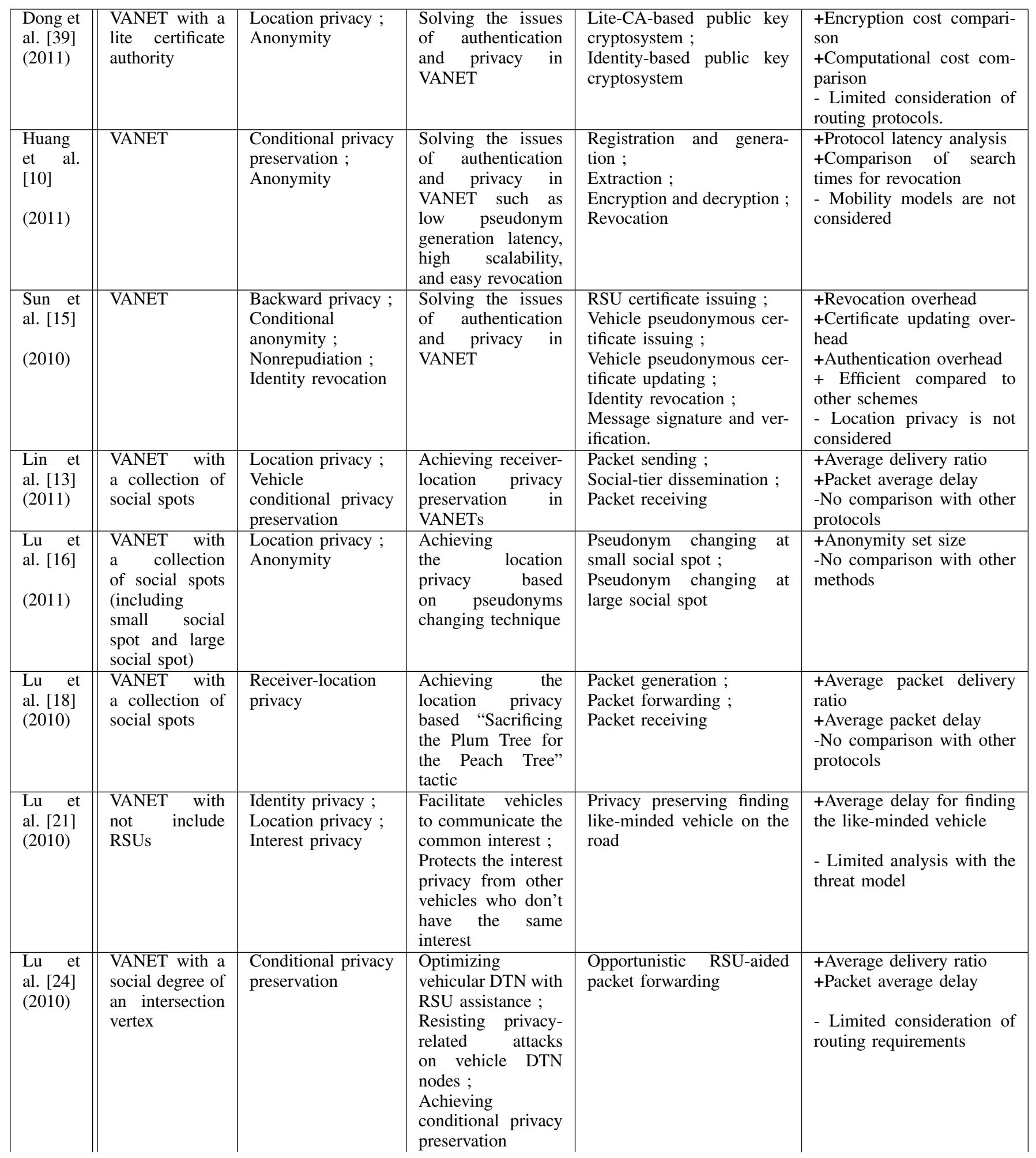




\begin{tabular}{|c|c|c|c|c|c|}
\hline $\begin{array}{l}\text { Li et al. } \\
\text { [9] } \\
\text { (2008) }\end{array}$ & VANET & $\begin{array}{l}\text { User privacy } \\
\text { preservation }\end{array}$ & $\begin{array}{l}\text { Achieving } \\
\text { conditional privacy } \\
\text { preservation based } \\
\text { on a lightweight } \\
\text { authenticated key } \\
\text { establishment } \\
\text { scheme }\end{array}$ & $\begin{array}{l}\text { Handling new } \\
\text { roadside devices, and } \\
\text { service providers ; } \\
\text { Scenario 1: } \\
\text { communications becure } \\
\text { vehicles; } \\
\begin{array}{l}\text { Scenario } 2: \\
\text { communications becure }\end{array} \\
\text { vehicles and roadside } \\
\text { devices; } \\
\begin{array}{l}\text { Scenario 3: a secure and } \\
\text { efficient communication }\end{array} \\
\text { scheme with privacy } \\
\text { preservation }\end{array}$ & $\begin{array}{l}\text { +Computational overhead } \\
\text { +Communication overhead } \\
\text { +Storage overhead } \\
\text { - Limited consideration of } \\
\text { routing requirements }\end{array}$ \\
\hline $\begin{array}{l}\text { Rabieh } \\
\text { et al. } \\
26\end{array}$ & $\begin{array}{l}\text { VANET with a } \\
\text { centralized au- } \\
\text { thority }\end{array}$ & Interest privacy & $\begin{array}{l}\text { Protects the interest } \\
\text { privacy from other } \\
\text { vehicles who don't } \\
\text { have the same inter- } \\
\text { est }\end{array}$ & $\begin{array}{l}\text { Chatting request packet; } \\
\text { Chatting response packet ; } \\
\text { Degree of interest verifica- } \\
\text { tion ; } \\
\text { Interest revocation }\end{array}$ & $\begin{array}{l}\text { +Computational overhead } \\
\text { +Communication overhead } \\
\text { - Limited analysis with the } \\
\text { threat model } \\
\text { - No comparison with other } \\
\text { protocols } \\
\text { - Mobility models are not } \\
\text { considered } \\
\text { - Location privacy is not } \\
\text { considered }\end{array}$ \\
\hline $\begin{array}{l}\text { Yu et } \\
\text { al. 29] } \\
(2016)\end{array}$ & $\begin{array}{l}\text { VANET with } \\
\text { data center and } \\
\text { a collection } \\
\text { of social spots } \\
\text { (including } \\
\text { Global Social } \\
\text { Spot and } \\
\text { Individual } \\
\text { Social Spot ) }\end{array}$ & Location privacy & $\begin{array}{l}\text { Exploit the meeting } \\
\text { opportunities } \\
\text { for pseudonym } \\
\text { changing; } \\
\text { Improve the } \\
\text { location privacy } \\
\text { preservation }\end{array}$ & $\begin{array}{l}\text { System initialization and } \\
\text { key generation ; } \\
\text { Group join ; } \\
\text { Pseudonyms exchanging ; } \\
\text { RSU signing protocol ; } \\
\text { Group leaving ; } \\
\text { Revocation protocol ; } \\
\text { Conditional tracking }\end{array}$ & $\begin{array}{l}\text { +Global pseudonym } \\
\text { entropy of the entire VSN } \\
\text { +Expected and actual } \\
\text { pseudonym entropy of } \\
\text { target vehicle } \\
+ \text { Comparison with existing } \\
\text { schemes } \\
+ \text { Analysis with the threat } \\
\text { model } \\
\text {-Many assumptions } \\
\text { needed to understand } \\
\text { implementation }\end{array}$ \\
\hline
\end{tabular}

TABLE XIII

MODELS OF SOCIAL SPOTS

\begin{tabular}{|l||l|l|}
\hline Scheme & Model & Idea \\
\hline $\begin{array}{l}\text { SPF protocol [18] (2010) } \\
\text { STAP protocol [13] (2011) }\end{array}$ & $\begin{array}{l}\text { Social spot integrated } \\
\text { with RSU }\end{array}$ & $\begin{array}{l}\text { Store packets in packet for- } \\
\text { warding }\end{array}$ \\
\hline $\begin{array}{l}\text { PCS strategy [28](2012) } \\
\text { Scheme [16] }(2011)\end{array}$ & $\begin{array}{l}\text { Small social spot and } \\
\text { large social spot }\end{array}$ & $\begin{array}{l}\text { Vehicle changes its pseudonym } \\
\text { at social spot }\end{array}$ \\
\hline MixGroup [29](2016) & $\begin{array}{l}\text { Global social spot and } \\
\text { individual social spot }\end{array}$ & $\begin{array}{l}\text { Exploit the meeting opportuni- } \\
\text { ties for pseudonym changing }\end{array}$ \\
\hline
\end{tabular}

\section{PRIVACY-PRESERVING SCHEMES FOR VSNS}

In this section, we in-detail examine nineteen privacy-preserving schemes developed for or applied in the context of VSNs. Based on the network model, we classify these schemes in three categories, including, social spots, location-based services, and service-oriented sociality. In addition, these schemes as shown in Tab. XII are published between 2008 and 2016.

\section{A. Network model with social spots}

The idea of placing social spots in VANET networks has emerged as an important research area, which is referred to as the locations where many vehicles will visit, for example, a sports complex, or a parking [103]. As shown in Tab. XIII] there are three models of social spots, including, 1) social spot integrated with RSU, 2) small social spot and large social spot, and 3) global social spot and individual social spot. In [28], the work developed a strategy called PCS, which consider the VANET with a collection of social spots in order to facilitate vehicles to achieve high-level location preservation. To preserving the location privacy, the PCS strategy uses three main phases, namely, key generation, pseudonym self-delegated generation, and conditional tracking. The PCS strategy is efficient in terms of anonymity set size and location privacy gain. In addition, the feasibility is proved using game-theoretic techniques but the authors conducted a limited analysis of the different threat models.

Similarly to the PCS strategy, Lin et al. in [13] developed a protocol called STAP. The STAP protocol considers social-tierassisted VANET network. With the assistance of social spot, STAP is not only very efficient in terms of packet delivery ratio 
and packet average delay, but also can preserves the location privacy. In order to unveil the asymptotic performance limits in MSN, the work in [104] can be applied in this category. In [16], Lu et al. proposed a social spot based pseudonyms changing technique, which considers VANET with a collection of social spots (including small social spot and large social spot). In addition, Lu et al. [16] proposed a protocol called SPF. The SPF protocol achieving the location privacy based "Sacrificing the Plum Tree for the Peach Tree" tactic. The SPF protocol is efficient in terms of average packet delivery ratio and average packet delay, but lacks comparison with similar protocols.

The MixGroup scheme in [29] is a recent interesting work, which considers a VANET with data center and a collection of social spots (including Global Social Spot and Individual Social Spot). With the assistance of social spot, MixGroup is not only very efficient for pseudonym changing, but also can improve the location privacy preservation of the users.

\section{B. Network model with location-based services}

The location-based services play an important role in ad hoc social networks [46], [42]. In [25], Lu et al. developed a scheme called DIKE, which considers a typical location-based service in VANET. Using the cooperative key update with $\mathrm{V}-2-\mathrm{V}$ communication, the DIKE scheme can preserve forward secrecy, backward secrecy, and collusion resistance. During each key update procedure, DIKE is efficient in terms of key update delay and key update ratio.

The applications services such as parking are very important in VANET [105], [106]. Lu et al. in [12] consider VANET with a large number of parking spaces. The work [12] developed an intelligent parking method that can be applied to large parking lots, which can support privacy-preserving of the drivers information. In addition, the work [12] is efficient in term of searching time delay. In a similar work Xiong et al. in [34] considering a VANET with a member manager, developed a protocol to support multi-level conditional privacy. This protocol is efficient in terms of storage requirements and computational overhead..

For solving the issues of authentication with privacy, Dong et al. in [39] proposed a scheme, called EP2DF, which considers VANET with a lite certificate authority. EP2DF is based on two cryptosystems, including, 1) lite-CA-based public key cryptosystem and 2) identity-based public key cryptosystem. The lite-CA-based public key cryptosystem is proposed specially to achieve lightweight public key certificate management. In addition, EP2DF is efficient in terms of encryption cost comparison and computational cost comparison. Related to EP2DF, Sun et al. [15] proposed a scheme called PASS, which it is efficient in terms of revocation overhead, certificate updating overhead, and authentication overhead. In addition, PASS is efficient compared to other schemes like ECPP scheme [60] and DCS scheme [107].

\section{Network model with service-oriented sociality}

The modeling of service-oriented sociality is based on a set of intersection nodes. Lu et al. in [24] proposed a scheme called SPRING and introduces the Social Degree of an intersection vertex in VANET, which is used for the optimal deployment of RSUs. In addition, the work in [24] proposes a method that optimizes vehicular DTN with RSU assistance. The SPRING scheme is efficient in terms of average delivery ratio and packet average delay. On the other hand, the FLIP scheme in [21] considers a VANET without the assistance of RSUs, which not only facilitates vehicles to communicate any common interest but can also protect the interest privacy from other vehicles who don't share the same interest. FLIP is efficient in terms of average delay for finding the like-minded vehicle.

Since the GPS integration into vehicles, each vehicle in a VANET can find the geographically shortest route based on a local map database [108]. Using social interplay, the NextCell scheme [109] can predict the location of a user from cell phone traces. However, obtaining an accurate position with privacy-preserving is very important for vehicle applications. Chim et al. in [20] proposed VSPN scheme, which can guide vehicles to desired destinations in a distributed manner and support privacy-preserving of the drivers. VSPN is efficient in terms of processing delay and reduction in travelling time.

Based on the VANET communications, Li et al. in [9] develop a schema called SECSPP and characterize the security in VANET by three scenarios, including, 1) secure communications between vehicles, 2) secure communications between vehicles and roadside devices, 3) a secure and efficient communication scheme with privacy preservation. SECSPP scheme is efficient in terms of computational overhead, communication overhead, and storage overhead. The scheme in [31] using a similar idea, proposed three authentication scenarios, namely, V2R and R2V authentication, V2V authentication, and cross-RSU V2V authentication.

Based on the state transition diagram in [10] for pseudonym generation, PACP scheme can solve the issues of authentication and privacy in VANET such as low pseudonym generation latency, high scalability, and easy revocation. In addition, PACP is efficient in terms of protocol latency analysis and comparison of search times for revocation. Related to PACP, SPECS scheme in [36] is efficient in terms of data transmission and invalid batch successful rate. On the other hand, the PPBMA scheme in [11] considers the link layer in privacy preserving broadcast message. PPBMA is efficient in terms of link layer delay and data packet delay. 
(a) Publications per year

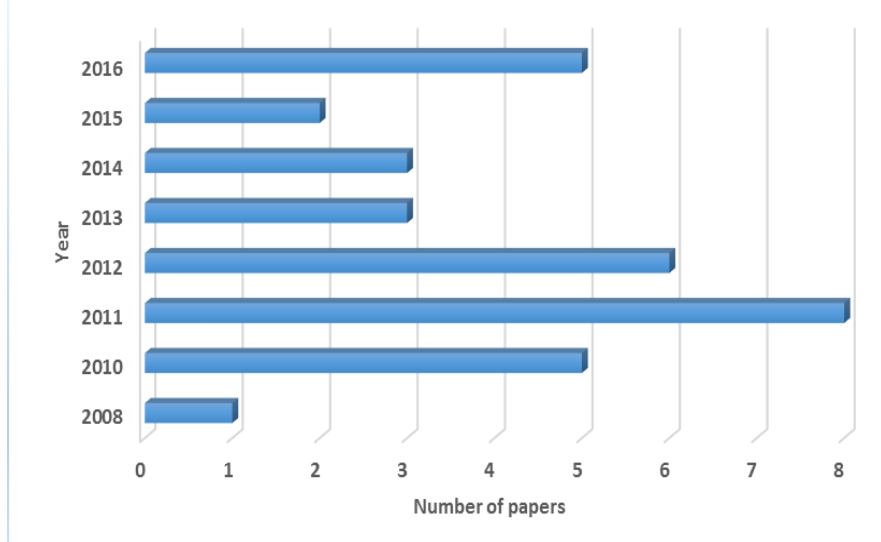

(c) Network Models

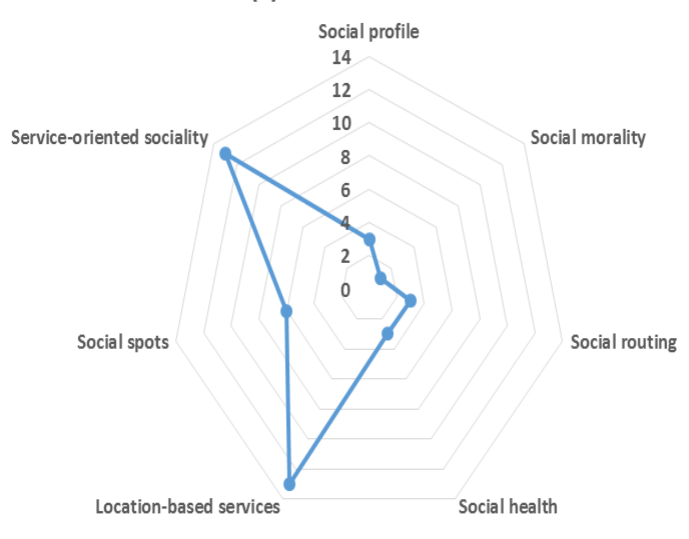

(b) Privacy models

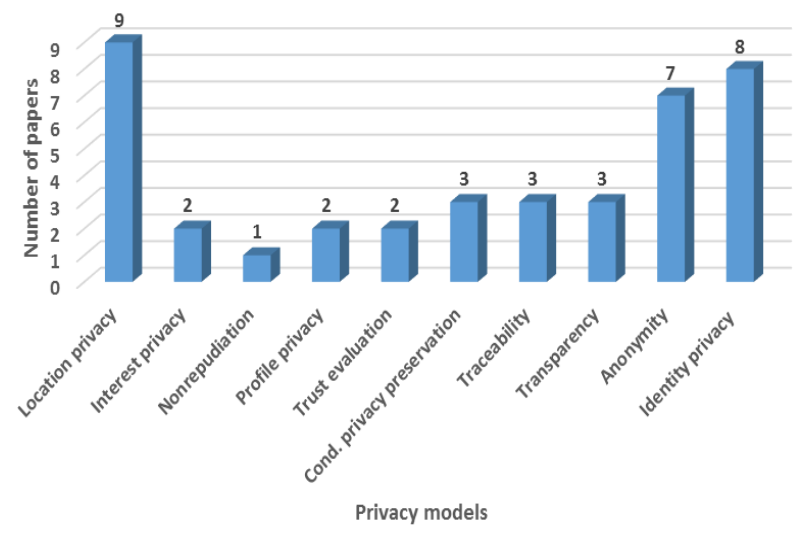

(d) Attacks, Countermeasures, Game theoretic approaches

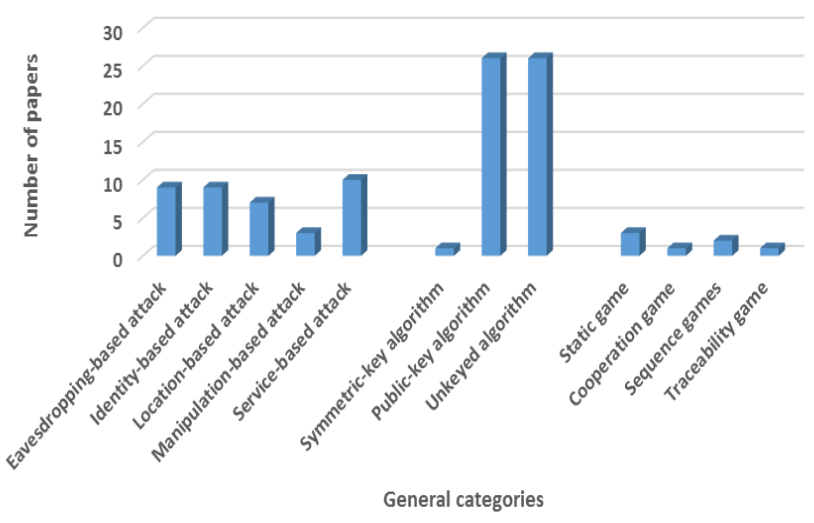

Fig. 7. (a) Publication per year, (b) Number of papers vs. privacy models, (c) Number of papers vs. network models, and (d) Number of papers vs. general categories

\section{RECOMMENDATIONS FOR FURTHER RESEARCH}

The average date of publication of the surveyed papers is 2012, as shown in Fig. 7)(a). However, 70\% of these papers focus on three privacy models, namely, location privacy, identity privacy, and anonymity, as shown in Fig. 77(b). For network models in MSNs and VSNs, most papers use service-oriented social, social spots, and location-based services, as shown in Fig. 7 (c). As shown in Fig. 7 (d), manipulation-based attacks are less considered and 95\% of the surveyed papers use both public-key algorithms and unkeyed algorithms. In addition, only seven papers use four types of game theory approach, namely, static game, cooperation game, sequence games, and traceability game.

In the remainder of this section, we will discuss four open issues for ad hoc social networks, including, privacy preserving methods, interdependent privacy, combination of privacy metrics, and identification of areas of vulnerability.

\section{A. Privacy-preserving methods}

We recommend three privacy-preserving methods, namely, 1) privacy-preserving energy consumption, 2) privacy preservation for V2G social networks, and 3) privacy preservation for social internet of vehicles.

- Privacy-preserving energy consumption: Privacy-preserving energy consumption is an open issue in ad hoc social networks. There are several research works addressing energy problems in ad hoc networks [110]. Based on optimal numbers of clusters, Ali et al. in [110] proposed an algorithm called MOPSO to manage the resources in order to make the MANET energy-efficient. A recent idea of Maglaras et al. in [111] can improve the use of social clusters based on semi-markov processes. Therefore, how to manage energy consumption under social clustering of nodes in ad hoc networks? Hence, privacy-preserving energy consumption is one of the future works.

- Privacy preservation for V2G social networks: A recent survey published in 2016 [112] review the state of the art of privacy-preserving schemes for V2G networks in smart grid, none of them carries study for the social characteristics in V2G networks. The future works addressing the limitations of privacy-preserving schemes for V2G networks will have an important contribution for $\mathrm{V} 2 \mathrm{G}$ social networks. 
- Privacy preservation for social internet of vehicles: Privacy preservation for social internet of vehicles (SIoV) is an open issue that we are working on [113]. Since the SIoV is a combination of social and vehicular networks, the future works addressing the limitations from both domains will have an important contribution for the SIoV.

\section{B. Interdepedent privacy}

Interdependent privacy refers to situations where the privacy of individual users is affected by the decisions of others [114]. Especially in social networks, where the interaction among different entities is constant and when talking for VSNs these entities are unknown [113] the interdependent privacy is playing a key role. One excellent example of privacy interdependence is the Facebook application platform. How well a user can protect his privacy from third party developers depends not only on his decisions, but also on the decisions of his friends. New applications that are based on the social concept of vehicular networking, like Navitweet [115] and Caravan Track [116] combined with traditional OSNs can create new privacy threats for the users.

\section{Combination of privacy metrics}

New privacy-enhancing technologies and those resulting from combinations of existing technologies need to be evaluated thoroughly to make sure that they provide an adequate amount of privacy. Because the VSN is a combination of social and vehicular networks, evaluations need to use a selection of privacy metrics from both domains [117].

\section{Identification of areas of vulnerability}

Since we are moving to the ear of IoT, a data breach from a system can be initiated from an attack that has occurred on another system that is somehow interconnected with it. For example in a VSN, an attack to a weak node can initiate malware propagation to the rest of the network, which is very difficult to detect and stop [118]. A smart vehicle with an out of date software [119] or an unprotected RSU can play the role of the weak node when an attack can be initiated. The US Department of Defense use the CARVER assessment method to determine criticality and vulnerability in enemy infrastructures. In a MSN/VSN the identification of critical nodes could help apply different privacy technologies according to how vulnerable each entity is. Therefore, research in vulnerability identification is needed in order to handle the complexity and heterogeneity of modern Ad Hoc social networks.

Although new sophisticated privacy metrics are needed, along with thorough analysis of the system in order to spot the weak players, no privacy metric is efficient if the human factor is neglected [120]. Organizations and society continue to be affected by both regular and similar cyber security breaches. These breaches pertain to technical implementations as well as routine processing of confidential electronic information. Despite this range of activities, it has been proven that half of these have human error at their core [121] and human aspects of cyber security must be taken into account when building new security and privacy mechanisms. Recently the idea of quantum cryptographic approaches for privacy preserving in cloud [122] and wireless systems [123] were introduced. Quantum cryptographic techniques provide an additional layer of security and privacy preservation of the system. Therefore, research in quantum privacy approaches is needed to handle the various attacks that MSNs and VSNs face. Although there exist simulator framework that combine many different simulators in order to better represent reality [124], the evaluation of the proposed metrics on real environments is also an open issue especially for VSNs where real deployments are limited.

\section{CONCLUSION}

In this article, we surveyed the state-of-the-art of privacy-preserving schemes for both MSNs and VSNs. We presented the major privacy models, including, location privacy, identity privacy, anonymity, traceability, interest privacy, backward privacy, and content oriented privacy. We also presented the major threats including, identity-based attacks, location-based attacks, eavesdropping-based attacks, manipulation-based attacks, and service-based attacks. We reviewed the countermeasures and game theoretic models proposed for MSN and VSN privacy preservation. We presented a side-by-side comparison in a tabular form for the current state-of-the-art of privacy-preserving schemes (thirty-three) proposed for MSN and VSN. Privacy preservation in MSNs and VSNs remains a challenging problem since adversaries can find different ways for exploiting vulnerabilites of the system. As we move to the IoT era, privacy preservation of a network cannot be treated in isolation but interedepedencies among users and networks must be taken into account. The correct identification of vulenrabilites of the system and the combincation of privacy metrics can improve the protection of the system, but no countermeasure can be effective if the human factor is neglected. 


\section{REFERENCES}

[1] Z. J. Haas, J. Deng, B. Liang, P. Papadimitratos, and S. Sajama, "Wireless ad hoc networks," Encyclopedia of Telecommunications, 2002.

[2] I. Chlamtac, M. Conti, and J. J.-N. Liu, "Mobile ad hoc networking: imperatives and challenges," Ad Hoc Networks, vol. 1, no. 1, pp. 13-64, jul 2003. [Online]. Available: http://linkinghub.elsevier.com/retrieve/pii/S1570870503000131

[3] S. Corson and J. Macker, "Mobile Ad hoc Networking (MANET): Routing Protocol Performance Issues and Evaluation Considerations," Computing Systems, vol. 54, pp. 1-12, 1999. [Online]. Available: http://www.ietf.org/rfc/rfc2501.txt

[4] M. Raya and J.-P. Hubaux, "Securing vehicular ad hoc networks," Journal of Computer Security, vol. 15, no. 1, pp. 39-68, jan 2007. [Online]. Available: http://www.medra.org/servlet/aliasResolver?alias=iospress $\{\&\}$ doi=10.3233/JCS-2007-15103

[5] K. Zhang, "Security and Privacy for Mobile Social Networks," Ph.D. dissertation, University of Waterloo, 2016.

[6] R. Lu, "Security and privacy preservation in vehicular social networks," Ph.D. dissertation, University of Waterloo, 2012.

[7] X. Liang, "Security and Privacy Preservation in Mobile Social Networks," Ph.D. dissertation, University of Waterloo, 2013.

[8] M. A. Ferrag, "La sécurisation des réseaux sociaux mobiles," Ph.D. dissertation, Badji Mokhtar-Annaba University, 2014.

[9] C.-T. Li, M.-S. Hwang, and Y.-P. Chu, "A secure and efficient communication scheme with authenticated key establishment and privacy preserving for vehicular ad hoc networks," Computer Communications, vol. 31, no. 12, pp. 2803-2814, jul 2008. [Online]. Available: http://linkinghub.elsevier.com/retrieve/pii/S0140366407005154

[10] D. Huang, S. Misra, M. Verma, and G. Xue, "PACP: An Efficient Pseudonymous Authentication-Based Conditional Privacy Protocol for VANETs," IEEE Transactions on Intelligent Transportation Systems, vol. 12, no. 3, pp. 736-746, sep 2011. [Online]. Available: http://ieeexplore.ieee.org/document/5982404/

[11] B. Ying, D. Makrakis, and H. T. Mouftah, "Privacy preserving broadcast message authentication protocol for VANETs," Journal of Network and Computer Applications, vol. 36, no. 5, pp. 1352-1364, sep 2013. [Online]. Available: http://linkinghub.elsevier.com/retrieve/pii/S1084804512001439]

[12] R. Lu, X. Lin, H. Zhu, and X. Shen, "An Intelligent Secure and Privacy-Preserving Parking Scheme Through Vehicular Communications," IEEE Transactions on Vehicular Technology, vol. 59, no. 6, pp. 2772-2785, jul 2010. [Online]. Available: http://ieeexplore.ieee.org/document/5456172/

[13] X. Lin, R. Lu, X. Liang, and X. Shen, "STAP: A social-tier-assisted packet forwarding protocol for achieving receiver-location privacy preservation in VANETs," in 2011 Proceedings IEEE INFOCOM. IEEE, apr 2011, pp. 2147-2155. [Online]. Available: http://ieeexplore.ieee.org/document/5935026/

[14] X. Liang, X. Lin, and X. S. Shen, "Enabling Trustworthy Service Evaluation in Service-Oriented Mobile Social Networks," IEEE Transactions on Parallel and Distributed Systems, vol. 25, no. 2, pp. 310-320, feb 2014. [Online]. Available: http://ieeexplore.ieee.org/document/6463403/

[15] Y. Sun, R. Lu, X. Lin, X. S. Shen, and J. Su, "An Efficient Pseudonymous Authentication Scheme With Strong Privacy Preservation for Vehicular Communications," IEEE Transactions on Vehicular Technology, vol. 59, no. 7, pp. 3589-3603, sep 2010. [Online]. Available: http://ieeexplore.ieee.org/document/5482136/

[16] R. Lu, X. Lin, T. H. Luan, X. Liang, and X. Shen, "Anonymity Analysis on Social Spot Based Pseudonym Changing for Location Privacy in VANETs," in 2011 IEEE International Conference on Communications (ICC). IEEE, jun 2011, pp. 1-5. [Online]. Available: http://ieeexplore.ieee.org/document/5962919/

[17] M. A. Ferrag, M. Nafa, and S. Ghanemi, "SDPP: an intelligent secure detection scheme with strong privacy-preserving for mobile peer-to-peer social network," International Journal of Information and Computer Security, vol. 6, no. 3, p. 241, 2014. [Online]. Available: http://www.inderscience.com/link.php?id=66650

[18] R. Lu, X. Lin, X. Liang, and X. Shen, "Sacrificing the Plum Tree for the Peach Tree: A Socialspot Tactic for Protecting Receiver-Location Privacy in VANET," in 2010 IEEE Global Telecommunications Conference GLOBECOM 2010. IEEE, dec 2010, pp. 1-5. [Online]. Available: http://ieeexplore.ieee.org/document/5683313/

[19] X. Liang, X. Li, T. H. Luan, R. Lu, X. Lin, and X. Shen, "Morality-Driven Data Forwarding With Privacy Preservation in Mobile Social Networks," IEEE Transactions on Vehicular Technology, vol. 61, no. 7, pp. 3209-3222, sep 2012. [Online]. Available: http://ieeexplore.ieee.org/document/6213569/

[20] T. W. Chim, S. M. Yiu, L. C. K. Hui, and V. O. K. Li, "VSPN: VANET-Based Secure and Privacy-Preserving Navigation," IEEE Transactions on Computers, vol. 63, no. 2, pp. 510-524, feb 2014. [Online]. Available: http://ieeexplore.ieee.org/document/6257366/]

[21] R. Lu, X. Lin, X. Liang, and X. Shen, "FLIP: An Efficient Privacy-Preserving Protocol for Finding Like-Minded Vehicles on the Road," in 2010 IEEE Global Telecommunications Conference GLOBECOM 2010. IEEE, dec 2010, pp. 1-5. [Online]. Available: http://ieeexplore.ieee.org/document/5684211/

[22] X. Liang, M. Barua, R. Lu, X. Lin, and X. S. Shen, "HealthShare: Achieving secure and privacy-preserving health information sharing through health social networks," Computer Communications, vol. 35, no. 15, pp. 1910-1920, sep 2012. [Online]. Available: http://linkinghub.elsevier.com/retrieve/pii/S0140366412000102

[23] K. Zhang, X. Liang, R. Lu, and X. Shen, "PIF: A Personalized Fine-Grained Spam Filtering Scheme With Privacy Preservation in Mobile Social Networks," IEEE Transactions on Computational Social Systems, vol. 2, no. 3, pp. 41-52, sep 2015. [Online]. Available: http://ieeexplore.ieee.org/document/7419280/

[24] R. Lu, X. Lin, and X. Shen, "SPRING: A Social-based Privacy-preserving Packet Forwarding Protocol for Vehicular Delay Tolerant Networks," in 2010 Proceedings IEEE INFOCOM. IEEE, mar 2010, pp. 1-9. [Online]. Available: http://ieeexplore.iee.org/document/5462161/

[25] R. Lu, X. Lin, X. Liang, and X. Shen, "A Dynamic Privacy-Preserving Key Management Scheme for Location-Based Services in VANETs," IEEE Transactions on Intelligent Transportation Systems, vol. 13, no. 1, pp. 127-139, mar 2012. [Online]. Available: http://ieeexplore.ieee.org/document/6012553/

[26] K. Rabieh, M. Mahmoud, A. Siraj, and J. Misic, "Efficient Privacy-Preserving Chatting Scheme with Degree of Interest Verification for Vehicular Social Networks," in 2015 IEEE Global Communications Conference (GLOBECOM). IEEE, dec 2015, pp. 1-6. [Online]. Available: http://ieeexplore.ieee.org/document/7417514/

[27] R. Lu, X. Lin, X. Liang, and X. Shen, "A Secure Handshake Scheme with Symptoms-Matching for mHealthcare Social Network," Mobile Networks and Applications, vol. 16, no. 6, pp. 683-694, dec 2011. [Online]. Available: http://link.springer.com/10.1007/s11036-010-0274-2

[28] R. Lu, X. Li, T. H. Luan, X. Liang, and X. Shen, "Pseudonym Changing at Social Spots: An Effective Strategy for Location Privacy in VANETs," IEEE Transactions on Vehicular Technology, vol. 61, no. 1, pp. 86-96, jan 2012. [Online]. Available: http://ieeexplore.ieee.org/document/5960806/

[29] R. Yu, J. Kang, X. Huang, S. Xie, Y. Zhang, and S. Gjessing, "MixGroup: Accumulative Pseudonym Exchanging for Location Privacy Enhancement in Vehicular Social Networks," IEEE Transactions on Dependable and Secure Computing, vol. 13, no. 1, pp. 93-105, jan 2016. [Online]. Available: http://ieexplore.iee.org/document/7042791/

[30] S. Hameed, X. Fu, P. Hui, and N. Sastry, "LENS: Leveraging social networking and trust to prevent spam transmission," in 2011 19th IEEE International Conference on Network Protocols. IEEE, oct 2011, pp. 13-18. [Online]. Available: http://ieeexplore.ieee.org/document/6089044/

[31] H. Lu, J. Li, and M. Guizani, "A novel ID-based authentication framework with adaptive privacy preservation for VANETs," in 2012 Computing, Communications and Applications Conference. IEEE, jan 2012, pp. 345-350. [Online]. Available: http://ieeexplore.ieee.org/document/6154869/

[32] H. Li, H. Zhu, S. Du, X. Liang, and X. Shen, "Privacy Leakage of Location Sharing in Mobile Social Networks: Attacks and Defense," IEEE Transactions on Dependable and Secure Computing, pp. 1-1, 2016. [Online]. Available: http://ieeexplore.iee.org/document/7556276/]

[33] Xiaohui Liang, Rongxing Lu, Le Chen, Xiaodong Lin, and Xuemin Shen, "PEC: A privacy-preserving emergency call scheme for mobile healthcare social networks," Journal of Communications and Networks, vol. 13, no. 2, pp. 102-112, apr 2011. [Online]. Available: http://ieeexplore.ieee.org/document/6157409/

[34] H. Xiong, Z. Chen, and F. Li, "Efficient and multi-level privacy-preserving communication protocol for VANET," Computers \& Electrical Engineering, vol. 38, no. 3, pp. 573-581, may 2012. [Online]. Available: http://linkinghub.elsevier.com/retrieve/pii/S0045790611001856 
[35] M. A. Ferrag, M. Nafa, and S. Ghanemi, "EPSA: an efficient and privacy-preserving scheme against wormhole attack on reactive routing for mobile ad hoc social networks," International Journal of Security and Networks, vol. 11, no. 3, p. 107, 2016. [Online]. Available: http://www.inderscience.com/link.php?id=78390

[36] T. Chim, S. Yiu, L. C. Hui, and V. O. Li, "SPECS: Secure and privacy enhancing communications schemes for VANETs," Ad Hoc Networks, vol. 9, no. 2, pp. 189-203, mar 2011. [Online]. Available: http://linkinghub.elsevier.com/retrieve/pii/S1570870510000648

[37] X. Liang, R. Lu, X. Lin, and X. Shen, "Profile Matching Protocol with Anonymity Enhancing Techniques," 2013, pp. 19-41. [Online]. Available: http://link.springer.com/10.1007/978-1-4614-8857-6\{_\}2

[38] X. Yang, R. Lu, H. Liang, and X. Tang, "SFPM: A Secure and Fine-Grained Privacy-Preserving Matching Protocol for Mobile Social Networking," Big Data Research, vol. 3, pp. 2-9, apr 2016. [Online]. Available: http://linkinghub.elsevier.com/retrieve/pii/S2214579615000507

[39] X. Dong, L. Wei, H. Zhu, Z. Cao, and L. Wang, "EP2DF: An Efficient Privacy-Preserving Data-Forwarding Scheme for Service-Oriented Vehicular Ad Hoc Networks," IEEE Transactions on Vehicular Technology, vol. 60, no. 2, pp. 580-591, feb 2011. [Online]. Available: http://ieeexplore.ieee.org/document/5645699/

[40] M. A. Ferrag, M. Nafa, and S. Ghanemi, "ECPDR: an efficient conditional privacy-preservation scheme with demand response for secure ad hoc social communications," International Journal of Embedded and Real-Time Communication Systems, vol. 4, no. 3, pp. 43-71, jan 2013. [Online]. Available: http://services.igi-global.com/resolvedoi/resolve.aspx?doi=10.4018/ijertcs.2013070103

[41] E. Luo, Q. Liu, J. H. Abawajy, and G. Wang, "Privacy-preserving multi-hop profile-matching protocol for proximity mobile social networks," Future Generation Computer Systems, oct 2016. [Online]. Available: http://linkinghub.elsevier.com/retrieve/pii/S0167739X16303107

[42] A. M. Vegni and V. Loscri, "A Survey on Vehicular Social Networks," IEEE Communications Surveys \& Tutorials, vol. 17, no. 4, pp. 2397-2419, jan 2015. [Online]. Available: http://ieeexplore.ieee.org/document/7151778/

[43] S. Al-Sultan, M. M. Al-Doori, A. H. Al-Bayatti, and H. Zedan, "A comprehensive survey on vehicular ad hoc network," Journal of network and computer applications, vol. 37, pp. 380-392, 2014.

[44] X. Liang, K. Zhang, X. Shen, and X. Lin, "Security and privacy in mobile social networks: challenges and solutions," IEEE Wireless Communications, vol. 21, no. 1, pp. 33-41, feb 2014. [Online]. Available: http://ieeexplore.ieee.org/document/6757895/

[45] J. H. Abawajy, M. I. H. Ninggal, and T. Herawan, "Privacy Preserving Social Network Data Publication," IEEE Communications Surveys \& Tutorials, vol. 18, no. 3, pp. 1974-1997, jan 2016. [Online]. Available: http://ieeexplore.ieee.org/document/7428813/

[46] X. Hu, T. H. S. Chu, V. C. M. Leung, E. C.-H. Ngai, P. Kruchten, and H. C. B. Chan, "A Survey on Mobile Social Networks: Applications, Platforms, System Architectures, and Future Research Directions," IEEE Communications Surveys \& Tutorials, vol. 17, no. 3, pp. 1557-1581, jan 2015. [Online]. Available: http://ieeexplore.ieee.org/lpdocs/epic03/wrapper.htm?arnumber=6960871

[47] J. Ni, K. Zhang, X. Lin, H. Yang, and X. S. Shen, "AMA: Anonymous mutual authentication with traceability in carpooling systems," in 2016 IEEE International Conference on Communications (ICC). IEEE, may 2016, pp. 1-6. [Online]. Available: http://ieeexplore.ieee.org/document/7511196/

[48] Jinyuan Sun, Chi Zhang, Yanchao Zhang, and Yuguang Fang, "SAT: A Security Architecture Achieving Anonymity and Traceability in Wireless Mesh Networks," IEEE Transactions on Dependable and Secure Computing, vol. 8, no. 2, pp. 295-307, mar 2011. [Online]. Available: http://ieeexplore.ieee.org/document/5374418/

[49] J. Sun, C. Zhang, and Y. Fang, "A Security Architecture Achieving Anonymity and Traceability in Wireless Mesh Networks," in IEEE INFOCOM 2008 The 27th Conference on Computer Communications. IEEE, apr 2008, pp. 1687-1695. [Online]. Available: http://ieeexplore.ieee.org/document/4509825/

[50] M. Li, H. Zhu, Z. Gao, S. Chen, L. Yu, S. Hu, and K. Ren, "All your location are belong to us," in Proceedings of the 15th ACM international symposium on Mobile ad hoc networking and computing - MobiHoc '14. New York, New York, USA: ACM Press, 2014, pp. 43-52. [Online]. Available: http://dl.acm.org/citation.cfm?doid=2632951.2632953

[51] X. Liang, Z. Cao, J. Shao, and H. Lin, "Short Group Signature Without Random Oracles," in International Conference on Information and Communications Security. Springer Berlin Heidelberg, 2007, pp. 69-82. [Online]. Available: http://link.springer.com/10.1007/978-3-540-77048-0\{_\}6

[52] G. Yan, D. B. Rawat, B. B. Bista, W. He, and A. Alnusair, "Privacy Protection in Vehicular Ad-Hoc Networks," in Transportation Systems and Engineering: Concepts, Methodologies, Tools, and Applications. Igi-global, 2015, pp. 272-309.

[53] L. A. Maglaras and D. Katsaros, "Enhanced spring clustering in vanets with obstruction considerations," in Vehicular Technology Conference (VTC Spring), 2013 IEEE 77th. IEEE, 2013, pp. 1-6.

[54] H. Chen, Y. Xiao, X. Hong, F. Hu, and J. L. Xie, "A survey of anonymity in wireless communication systems," Security and Communication Networks, vol. 2, no. 5, pp. 427-444, 2009.

[55] L. SWEENEY, "k-ANONYMITY: A MODEL FOR PROTECTING PRIVACY," International Journal of Uncertainty, Fuzziness and Knowledge-Based Systems, vol. 10, no. 05, pp. 557-570, oct 2002. [Online]. Available: http://www.worldscientific.com/doi/abs/10.1142/S0218488502001648

[56] L. von Ahn, A. Bortz, and N. J. Hopper, "k-anonymous message transmission," in Proceedings of the 10th ACM conference on Computer and communication security - CCS '03. New York, New York, USA: ACM Press, 2003, p. 122. [Online]. Available: http://portal.acm.org/citation.cfm?doid=948109.948128

[57] P. Wang, P. Ning, and D. S. Reeves, "A k -anonymous communication protocol for overlay networks," in Proceedings of the 2nd ACM symposium on Information, computer and communications security - ASIACCS '07. New York, New York, USA: ACM Press, 2007, p. 45. [Online]. Available: http://portal.acm.org/citation.cfm?doid=1229285.1229296

[58] H. Shacham and B. Waters, "Efficient Ring Signatures Without Random Oracles," in Public Key Cryptography PKC $2007 . \quad$ Berlin, Heidelberg: Springer Berlin Heidelberg, pp. 166-180. [Online]. Available: http://link.springer.com/10.1007/978-3-540-71677-8\{_\}12

[59] R. L. Rivest, A. Shamir, and Y. Tauman, "How to Leak a Secret," in International Conference on the Theory and Application of Cryptology and Information Security. Springer Berlin Heidelberg, 2001, pp. 552-565. [Online]. Available: http://link.springer.com/10.1007/3-540-45682-1\{_\}32

[60] R. Lu, X. Lin, H. Zhu, P.-H. Ho, and X. Shen, "ECPP: Efficient Conditional Privacy Preservation Protocol for Secure Vehicular Communications," in IEEE INFOCOM 2008 - The 27th Conference on Computer Communications. IEEE, apr 2008, pp. 1229-1237. [Online]. Available: http://ieeexplore.ieee.org/document/4509774/

[61] X. Boyen and B. Waters, "Full-Domain Subgroup Hiding and Constant-Size Group Signatures," in Public Key Cryptography PKC 2007. Berlin, Heidelberg: Springer Berlin Heidelberg, pp. 1-15. [Online]. Available: http://link.springer.com/10.1007/978-3-540-71677-8\{_\}1

[62] D. Chaum, "Blind Signature System," in Advances in Cryptology. Boston, MA: Springer US, 1984, pp. 153-153. [Online]. Available: http://link.springer.com/10.1007/978-1-4684-4730-9\{_\}14

[63] A. Juels, M. Luby, and R. Ostrovsky, "Security of blind digital signatures," in Annual International Cryptology Conference. Springer Berlin Heidelberg, 1997, pp. 150-164. [Online]. Available: http://link.springer.com/10.1007/BFb0052233

[64] M. Bellare, D. Pointcheval, and P. Rogaway, "Authenticated Key Exchange Secure against Dictionary Attacks," in International Conference on the Theory and Applications of Cryptographic Techniques. Springer Berlin Heidelberg, 2000, pp. 139-155. [Online]. Available: http://link.springer.com/10.1007/3-540-45539-6\{_\}11

[65] B. Wu, J. Chen, J. Wu, and M. Cardei, "A Survey of Attacks and Countermeasures in Mobile Ad Hoc Networks," in Wireless Network Security. Boston, MA: Springer US, pp. 103-135. [Online]. Available: http://link.springer.com/10.1007/978-0-387-33112-6\{_\}5

[66] M. A. Ferrag, M. Nafa, and S. Ghanemi, "Security and Privacy in Mobile Ad hoc Social Networks," in Security, Privacy, Trust, and Resource Management in Mobile and Wireless Communications. IGI Global, pp. 222-243. [Online]. Available: http://services.igi-global.com/resolvedoi/resolve. aspx?doi=10.4018/978-1-4666-4691-9.ch010

[67] R. Akbani, T. Korkmaz, and G. V. S. Raju, "Mobile Ad-Hoc Networks Security," in Recent Advances in Computer Science and Information Engineering. Springer Berlin Heidelberg, 2012, pp. 659-666. [Online]. Available: http://link.springer.com/10.1007/978-3-642-25769-8\{_\}92 
[68] M. A. Ferrag, N. Chekkai, and M. Nafa, "Securing Embedded Systems: Cyberattacks, Countermeasures, and Challenges," in Securing Cyber-Physical Systems. CRC Press, oct 2015, pp. 279-304. [Online]. Available: http://www.crcnetbase.com/doi/10.1201/b19311-12

[69] T. Zhou, R. R. Choudhury, P. Ning, and K. Chakrabarty, "Privacy-Preserving Detection of Sybil Attacks in Vehicular Ad Hoc Networks," in 2007 Fourth Annual International Conference on Mobile and Ubiquitous Systems: Networking \& Services (MobiQuitous). IEEE, 2007, pp. 1-8. [Online]. Available: http://ieeexplore.ieee.org/document/4451013/

[70] M. Conti, N. Dragoni, and V. Lesyk, "A Survey of Man In The Middle Attacks," IEEE Communications Surveys \& Tutorials, vol. 18, no. 3, pp. 2027-2051, jan 2016. [Online]. Available: http://ieeexplore.ieee.org/document/7442758/

[71] B. Preneel, "The State of Cryptographic Hash Functions," in Lectures on Data Security. Springer Berlin Heidelberg, 1999, pp. 158-182. [Online]. Available: http://link.springer.com/10.1007/3-540-48969-X\{_\}8

[72] C. Gentry, "a Fully Homomorphic Encryption Scheme," Ph.D. dissertation, Stanford University, 2009. [Online]. Available: papers2: //publication/uuid/894E7CEA-E13A-4034-9EC1-317B0D47C79D

[73] J. Petit, F. Schaub, M. Feiri, and F. Kargl, "Pseudonym Schemes in Vehicular Networks: A Survey," IEEE Communications Surveys \& Tutorials, vol. 17, no. 1, pp. 228-255, jan 2015. [Online]. Available: http://ieeexplore.ieee.org/lpdocs/epic03/wrapper.htm?arnumber=6873216

[74] H. Krawczyk, M. Bellare, and R. Canetti, "RFC2104 - HMAC: Keyed-hashing for message authentication," Tech. Rep., 1997.

[75] D. Boneh and X. Boyen, "Short Signatures Without Random Oracles and the SDH Assumption in Bilinear Groups," Journal of Cryptology, vol. 21, no. 2, pp. 149-177, apr 2008. [Online]. Available: http://link.springer.com/10.1007/s00145-007-9005-7

[76] C. Gentry and Z. Ramzan, "Identity-Based Aggregate Signatures," in International Workshop on Public Key Cryptography. Springer Berlin Heidelberg, 2006, pp. 257-273. [Online]. Available: http://link.springer.com/10.1007/11745853\{_\}17

[77] D. Boneh and M. Franklin, "Identity-Based Encryption from the Weil Pairing," in Annual International Cryptology Conference. Springer Berlin Heidelberg, 2001, pp. 213-229. [Online]. Available: http://link.springer.com/10.1007/3-540-44647-8\{_\}13

[78] D. Boneh, G. Di Crescenzo, R. Ostrovsky, and G. Persiano, "Public Key Encryption with Keyword Search," in International Conference on the Theory and Applications of Cryptographic Techniques. Springer Berlin Heidelberg, 2004, pp. 506-522. [Online]. Available: http://link.springer.com/10.1007/978-3-540-24676-3\{_\}30

[79] C. Bösch, P. Hartel, W. Jonker, and A. Peter, "A Survey of Provably Secure Searchable Encryption," ACM Computing Surveys, vol. 47, no. 2, pp. 1-51, aug 2014. [Online]. Available: http://dl.acm.org/citation.cfm?doid=2658850.2636328

[80] A. Beimel, A. Ben-Efraim, C. Padró, and I. Tyomkin, "Multi-linear Secret-Sharing Schemes," in Theory of Cryptography Conference. Springer Berlin Heidelberg, 2014, pp. 394-418. [Online]. Available: http://link.springer.com/10.1007/978-3-642-54242-8\{_\}17

[81] S. Hohenberger and B. Waters, "Attribute-Based Encryption with Fast Decryption," in Public-Key CryptographyPKC 2013. Springer Berlin Heidelberg, 2013, pp. 162-179. [Online]. Available: http://link.springer.com/10.1007/978-3-642-36362-7\{_\}11

[82] V. Iovino and G. Persiano, "Hidden-Vector Encryption with Groups of Prime Order," in Pairing-Based Cryptography Pairing 2008. Berlin, Heidelberg: Springer Berlin Heidelberg, pp. 75-88. [Online]. Available: http://link.springer.com/10.1007/978-3-540-85538-5\{_\}5

[83] J. H. Park, "Efficient Hidden Vector Encryption for Conjunctive Queries on Encrypted Data," IEEE Transactions on Knowledge and Data Engineering, vol. 23, no. 10, pp. 1483-1497, oct 2011. [Online]. Available: http://ieeexplore.ieee.org/document/5611518/

[84] D. Boneh and H. Shacham, "Group signatures with verifier-local revocation," in Proceedings of the 11th ACM conference on Computer and communications security - CCS '04. New York, New York, USA: ACM Press, 2004, p. 168. [Online]. Available: http://portal.acm.org/citation.cfm?doid=1030083.1030106

[85] L. Law, A. Menezes, M. Qu, J. Solinas, and S. Vanstone, "An efficient protocol for authenticated key agreement," Designs, Codes and Cryptography, vol. 28, no. 2, pp. 119-134, 2003. [Online]. Available: http://link.springer.com/10.1023/A:1022595222606

[86] M. Green and G. Ateniese, "Identity-Based Proxy Re-encryption," in Applied Cryptography and Network Security. Springer Berlin Heidelberg, 2007, pp. 288-306. [Online]. Available: http://link.springer.com/10.1007/978-3-540-72738-5\{_\}19

[87] A. Shamir, "Identity-Based Cryptosystems and Signature Schemes," in Advances in Cryptology. Springer Berlin Heidelberg, 1984, pp. 47-53. [Online]. Available: http://link.springer.com/10.1007/3-540-39568-7\{_\}5

[88] A. Shamir and Y. Tauman, "Improved Online/Offline Signature Schemes," in Annual International Cryptology Conference. Springer Berlin Heidelberg, 2001, pp. 355-367. [Online]. Available: http://link.springer.com/10.1007/3-540-44647-8\{_\}21

[89] S. S. Al-Riyami and K. G. Paterson, "Certificateless Public Key Cryptography," in International Conference on the Theory and Application of Cryptology and Information Security. Springer Berlin Heidelberg, 2003, pp. 452-473. [Online]. Available: http://link.springer.com/10.1007/ 978-3-540-40061-5\{_- 29

[90] — "CBE from CL-PKE: A Generic Construction and Efficient Schemes," in In International Workshop on Public Key Cryptography. Springer Berlin Heidelberg, 2005, pp. 398-415. [Online]. Available: http://link.springer.com/10.1007/978-3-540-30580-4\{_\}27

[91] C. Schnorr, "Efficient signature generation by smart cards," Journal of Cryptology, vol. 4, no. 3, 1991. [Online]. Available: http: //link.springer.com/10.1007/BF00196725

[92] D. Johnson, A. Menezes, and S. Vanstone, "The Elliptic Curve Digital Signature Algorithm (ECDSA)," International Journal of Information Security, vol. 1, no. 1, pp. 36-63, aug 2001. [Online]. Available: http://link.springer.com/10.1007/s102070100002

[93] U. M. Maurer and Y. Yacobi, "Non-interactive Public-Key Cryptography," in Advances in Cryptology EUROCRYPT '91. Berlin, Heidelberg: Springer Berlin Heidelberg, pp. 498-507. [Online]. Available: http://link.springer.com/10.1007/3-540-46416-6\{_\}43

[94] K.-A. Shim, "A Survey of Public-Key Cryptographic Primitives in Wireless Sensor Networks," IEEE Communications Surveys \& Tutorials, vol. 18, no. 1, pp. 577-601, jan 2016. [Online]. Available: http://ieeexplore.ieee.org/document/7172449/

[95] M. H. Manshaei, Q. Zhu, T. Alpcan, T. Bacar, and J.-P. Hubaux, "Game theory meets network security and privacy," ACM Computing Surveys, vol. 45, no. 3, pp. 1-39, jun 2013. [Online]. Available: http://dl.acm.org/citation.cfm?doid=2480741.2480742

[96] X. Liang and Y. Xiao, "Game Theory for Network Security," IEEE Communications Surveys \& Tutorials, vol. 15, no. 1, pp. 472-486, jan 2013. [Online]. Available: http://ieeexplore.ieee.org/document/6238282/

[97] N. Sastry and D. Wagner, "Security considerations for IEEE 802.15.4 networks," in Proceedings of the 2004 ACM workshop on Wireless security WiSe '04. New York, New York, USA: ACM Press, 2004, p. 32. [Online]. Available: http://portal.acm.org/citation.cfm?doid=1023646.1023654

[98] R. Rothblum, "Homomorphic Encryption: From Private-Key to Public-Key," in Theory of Cryptography Conference. Springer Berlin Heidelberg, 2011, pp. 219-234. [Online]. Available: http://link.springer.com/10.1007/978-3-642-19571-6\{_\}14

[99] J. Freudiger, M. H. Manshaei, J.-P. Hubaux, and D. C. Parkes, "On non-cooperative location privacy," in Proceedings of the 16th ACM conference on Computer and communications security - CCS '09. New York, New York, USA: ACM Press, 2009, p. 324. [Online]. Available: http://portal.acm.org/citation.cfm?doid=1653662.1653702

[100] T. Clausen and P. Jacquet, "Optimized Link State Routing Protocol (OLSR)," Ietf Rfc3626, p. 75, 2003.

[101] C. Perkings, E. Belding-Royer, and S. Das, "Ad hoc On-Demand Distance Vector (AODV) Routing," Ietf Rfc 3561, pp. 1-37, 2003. [Online]. Available: http://tools.ietf.org/pdf/rfc3561.pdf

[102] Kuan Zhang, X. Liang, R. Lu, X. Shen, and Hai Zhao, "VSLP: Voronoi-socialspot-aided packet forwarding protocol with receiver Location Privacy in MSNs," in 2012 IEEE Global Communications Conference (GLOBECOM). IEEE, dec 2012, pp. 348-353. [Online]. Available: http://ieeexplore.ieee.org/document/6503137/

[103] Z. Su, Y. Hui, and S. Guo, "D2D-based content delivery with parked vehicles in vehicular social networks," IEEE Wireless Communications, vol. 23, no. 4, pp. 90-95, aug 2016. [Online]. Available: http://ieeexplore.ieee.org/document/7553031/ 
[104] N. Lu, T. H. Luan, M. Wang, X. Shen, and F. Bai, "Bounds of Asymptotic Performance Limits of Social-Proximity Vehicular Networks," IEEE/ACM Transactions on Networking, vol. 22, no. 3, pp. 812-825, jun 2014. [Online]. Available: http://ieeexplore.ieee.org/document/6515701/

[105] M. Caliskan, D. Graupner, and M. Mauve, "Decentralized discovery of free parking places," in Proceedings of the 3rd international workshop on Vehicular ad hoc networks - VANET '06. New York, New York, USA: ACM Press, 2006, p. 30. [Online]. Available: http://portal.acm.org/citation.cfm?doid=1161064.1161070

[106] R. Panayappan, J. M. Trivedi, A. Studer, and A. Perrig, "VANET-based approach for parking space availability," in Proceedings of the fourth ACM international workshop on Vehicular ad hoc networks - VANET '07. New York, New York, USA: ACM Press, 2007, p. 75. [Online]. Available: http://portal.acm.org/citation.cfm?doid=1287748.1287763

[107] A. Wasef, Yixin Jiang, and Xuemin Shen, "DCS: An Efficient Distributed-Certificate-Service Scheme for Vehicular Networks," IEEE Transactions on Vehicular Technology, vol. 59, no. 2, pp. 533-549, feb 2010. [Online]. Available: http://ieeexplore.ieee.org/document/5184904/

[108] J. Liu, J. Wan, Q. Wang, P. Deng, K. Zhou, and Y. Qiao, “A survey on position-based routing for vehicular ad hoc networks," Telecommunication Systems, vol. 62, no. 1, pp. 15-30, may 2016. [Online]. Available: http://link.springer.com/10.1007/s11235-015-9979-7

[109] D. Zhang, D. Zhang, H. Xiong, L. T. Yang, and V. Gauthier, "NextCell: Predicting Location Using Social Interplay from Cell Phone Traces," IEEE Transactions on Computers, vol. 64, no. 2, pp. 452-463, feb 2015. [Online]. Available: http://ieeexplore.ieee.org/document/6671589/

[110] H. Ali, W. Shahzad, and F. A. Khan, "Energy-efficient clustering in mobile ad-hoc networks using multi-objective particle swarm optimization," Applied Soft Computing, vol. 12, no. 7, pp. 1913-1928, jul 2012. [Online]. Available: http://linkinghub.elsevier.com/retrieve/pii/S1568494611001967

[111] L. A. Maglaras and D. Katsaros, "Social Clustering of Vehicles Based on Semi-Markov Processes," IEEE Transactions on Vehicular Technology, vol. 65, no. 1, pp. 318-332, jan 2016. [Online]. Available: http://ieeexplore.ieee.org/document/7015614/

[112] W. Han and Y. Xiao, "Privacy preservation for V2G networks in smart grid: A survey," Computer Communications, vol. 91-92, pp. 17-28, oct 2016. [Online]. Available: http://linkinghub.elsevier.com/retrieve/pii/S0140366416302572

[113] L. Maglaras, A. Al-Bayatti, Y. He, I. Wagner, and H. Janicke, "Social Internet of Vehicles for Smart Cities," Journal of Sensor and Actuator Networks, vol. 5, no. 1, p. 3, feb 2016. [Online]. Available: http://www.mdpi.com/2224-2708/5/1/3

[114] G. Biczók and P. H. Chia, "Interdependent Privacy: Let Me Share Your Data," in International Conference on Financial Cryptography and Data Security. Springer Berlin Heidelberg, 2013, pp. 338-353. [Online]. Available: http://link.springer.com/10.1007/978-3-642-39884-1\{_\}29

[115] W. Sha, D. Kwak, B. Nath, and L. Iftode, "Social vehicle navigation," in Proceedings of the 14th Workshop on Mobile Computing Systems and Applications - HotMobile '13. New York, New York, USA: ACM Press, 2013, p. 1. [Online]. Available: http: //dl.acm.org/citation.cfm?doid=2444776.2444798

[116] C. Squatriglia, "Ford's Tweeting Car Embarks on American Journey 2.0. Wired," 2010. [Online]. Available: https://www.wired.com/2010/05/ ford-american-journey/

[117] I. Wagner and D. Eckhoff, "Technical Privacy Metrics: a Systematic Survey,” dec 2015. [Online]. Available: http://arxiv.org/abs/1512.00327

[118] P. Basaras, I. Belikaidis, L. Maglaras, and D. Katsaros, "Blocking epidemic propagation in vehicular networks," in 2016 12th Annual Conference on Wireless On-demand Network Systems and Services (WONS). IEEE, 2016, pp. 1-8.

[119] H. Onishi, "Approaches for vehicle cyber security," in 2014 IEEE Conference on Communications and Network Security. IEEE, oct 2014, pp. 506-507. [Online]. Available: http://ieeexplore.ieee.org/lpdocs/epic03/wrapper.htm?arnumber=6997527

[120] M. Evans, L. A. Maglaras, Y. He, and H. Janicke, "Human Behaviour as an aspect of Cyber Security Assurance," jan 2016. [Online]. Available: http://arxiv.org/abs/1601.03921

[121] "Information Security Breaches Survey," 2015. [Online]. Available: https://www.gov.uk/government/uploads/system/uploads/attachment $\left\{\begin{array}{c}\text { \} } \\ \text { data/file/ }\end{array}\right.$ 432412/bis-15-302-information $\left\{_{-}\right\}$security $\left\{_{-}\right\}$breaches $\left\{_{-}\right\}$survey $\left\{_{-}\right\} 2015$-full-report.pdf

[122] K. Vidya, V. Alamelu, K. S. Kumar, and L. S. Chandraa, "Quantum cryptographic approach to decentralized access control and privacy preserving in cloud," Asian Journal of Information Technology, vol. 15, no. 3, pp. 578-592, 2016.

[123] R. Nomula, M. E. Rifai, and P. Verma, "Multi-photon tolerant protocols for quantum secure communication in wireless standards," International Journal of Security and Networks, vol. 11, no. 1-2, pp. 25-36, 2016.

[124] D. Kosmanos, N. Prodromou, A. Argyriou, L. Maglaras, and H. Janicke, "Mimo techniques for improving diversity and suppressing jamming threats in vehicular ad hoc networks," Mobile Information Systems, 2016. 